\title{
Nonlinear Langevin Equation of Hadamard-Caputo Type Fractional Derivatives with Nonlocal Fractional Integral Conditions
}

\author{
Jessada Tariboon, ${ }^{1}$ Sotiris K. Ntouyas, ${ }^{2,3}$ and Chatthai Thaiprayoon ${ }^{1}$ \\ ${ }^{1}$ Nonlinear Dynamic Analysis Research Center, Department of Mathematics, Faculty of Applied Science, \\ King Mongkut's University of Technology North Bangkok, Bangkok 10800, Thailand \\ ${ }^{2}$ Department of Mathematics, University of Ioannina, 45110 Ioannina, Greece \\ ${ }^{3}$ Nonlinear Analysis and Applied Mathematics Research Group (NAAM), Department of Mathematics, Faculty of Science, \\ King Abdulaziz University, P.O. Box 80203, Jeddah 21589, Saudi Arabia
}

Correspondence should be addressed to Jessada Tariboon; jessadat@kmutnb.ac.th

Received 27 August 2014; Accepted 21 October 2014; Published 26 November 2014

Academic Editor: Andrei D. Mironov

Copyright ( $(2014$ Jessada Tariboon et al. This is an open access article distributed under the Creative Commons Attribution License, which permits unrestricted use, distribution, and reproduction in any medium, provided the original work is properly cited.

We study existence and uniqueness of solutions for a problem consisting of nonlinear Langevin equation of Hadamard-Caputo type fractional derivatives with nonlocal fractional integral conditions. A variety of fixed point theorems are used, such as Banach's fixed point theorem, Krasnoselskii's fixed point theorem, Leray-Schauder's nonlinear alternative, and Leray-Schauder's degree theory. Enlightening examples illustrating the obtained results are also presented.

\section{Introduction}

In this paper, we study the existence and uniqueness of solutions for nonlinear Langevin equation of HadamardCaputo type fractional derivatives with nonlocal fractional integral conditions of the form

$$
\begin{gathered}
D^{\alpha}\left(D^{\beta}+\lambda\right) x(t)=f(t, x(t)), \quad 1<t<e, \\
\sum_{i=1}^{m} \theta_{i} I^{\mu_{i}} x\left(\eta_{i}\right)=\sum_{j=1}^{n} \phi_{j} I^{\gamma_{j}} x\left(\omega_{j}\right), \\
\sum_{k=1}^{p} \varepsilon_{k} I^{\sigma_{k}} x\left(\psi_{k}\right)=\sum_{l=1}^{q} \nu_{l} I^{\tau_{l}} x\left(\varphi_{l}\right),
\end{gathered}
$$

where $D^{\rho}$ denotes the Caputo-type Hadamard fractional derivative of order $\rho, \rho=\{\alpha, \beta\}$ with $0<\alpha, \beta<1,1<$ $\alpha+\beta<2, \lambda$ is a given constant, $f:[1, e] \times \mathbb{R} \rightarrow \mathbb{R}$ is a continuous function, $I^{\kappa}$ is the Hadamard fractional integral of order $\kappa>0, \kappa=\left\{\mu_{i}, \gamma_{j}, \sigma_{k}, \tau_{l}\right\}$, the constants $\eta_{i}, \omega_{j}, \psi_{k}$, $\varphi_{l} \in(1, e)$, and $\theta_{i}, \phi_{j}, \varepsilon_{k}, v_{l} \in \mathbb{R}$, for all $i=1,2, \ldots, m$, $j=1,2, \ldots, n, k=1,2, \ldots, p$, and $l=1,2, \ldots, q$.

The significance of investigating nonlocal problem (1) is that the nonlocal Hadamard fractional integral conditions do not contain boundary values of unknown function $x$, which is a new novel for studying nonboundary problems. In particular, if $m=n=p=q=1$, then the conditions of (1) are reduced to

$$
\begin{aligned}
& \frac{\theta_{1}}{\Gamma\left(\mu_{1}\right)} \int_{1}^{\eta_{1}}\left(\log \frac{t}{s}\right)^{\mu_{1}-1} \frac{x(s)}{s} d s \\
& =\frac{\phi_{1}}{\Gamma\left(\gamma_{1}\right)} \int_{1}^{\omega_{1}}\left(\log \frac{t}{s}\right)^{\gamma_{1}-1} \frac{x(s)}{s} d s, \\
& \frac{\varepsilon_{1}}{\Gamma\left(\sigma_{1}\right)} \int_{1}^{\psi_{1}}\left(\log \frac{t}{s}\right)^{\sigma_{1}-1} \frac{x(s)}{s} d s \\
& =\frac{\nu_{1}}{\Gamma\left(\tau_{1}\right)} \int_{1}^{\varphi_{1}}\left(\log \frac{t}{s}\right)^{\tau_{1}-1} \frac{x(s)}{s} d s,
\end{aligned}
$$

where $\log (\cdot)=\log _{e}(\cdot)$. 
The subject of fractional differential equations has recently evolved as an interesting and popular field of research. In fact, fractional derivatives provide an excellent tool for the description of memory and hereditary properties of various materials and processes. More and more researchers have found that fractional differential equations play important roles in many research areas, such as physics, chemical technology, population dynamics, biotechnology, and economics. For examples and recent development of the topic, see [1-13]. However, it has been observed that most of the work on the topic involves either RiemannLiouville or Caputo-type fractional derivative. Besides these derivatives, Hadamard fractional derivative is another kind of fractional derivatives that was introduced by Hadamard in 1892 [14]. This fractional derivative differs from the other ones in the sense that the kernel of the integral (in the definition of Hadamard derivative) contains logarithmic function of arbitrary exponent. For background material of Hadamard fractional derivative and integral, we refer to the papers [3, 15-19].

The Langevin equation (first formulated by Langevin in 1908 ) is found to be an effective tool to describe the evolution of physical phenomena in fluctuating environments [20]. For some new developments on the fractional Langevin equation, see, for example, [21-29].

Differential equations with nonlocal boundary conditions are often used to take into account some peculiarities of physical, chemical, biological, or other processes, which is impossible by applying classical boundary conditions. Nonlocal problem with integral boundary condition instead of classical Dirichlet or Neumann boundary condition for one-dimensional parabolic equation first was considered by Cannon [30] to describe the process of heat conduction, which was reduced to the integral equation and investigated in the spaces of classical functions. Nonlocal integral boundary conditions can be used when it is impossible to directly determine the values of the sought quantity on the boundary and we know its total amount or integral average on space domain, for example, total energy, average temperature, and total mass of impurities. Some results on problems with nonlocal integral boundary conditions for various evolution equations were investigated; for example, see [31-34].

Several new existence and uniqueness results are proved by using a variety of fixed point theorems (such as Banach contraction principle, Krasnoselskii's fixed point theorem, Leray-Schauder's nonlinear alternative, and Leray-Schauder's degree theory).

The rest of the paper is organized as follows. In Section 2, we recall some preliminary facts that we need in the sequel. In Section 3, we present our existence and uniqueness results. Examples illustrating the obtained results are presented in Section 4 .

\section{Preliminaries}

In this section, we introduce some notations and definitions of fractional calculus $[2,3]$ and present preliminary results needed in our proofs later.
Definition 1. For at least $n$-times differentiable function $g$ : $[1, \infty) \rightarrow \mathbb{R}$, the Caputo-type Hadamard derivative of fractional order $\alpha$ is defined as

$$
\begin{array}{r}
D^{\alpha} g(t)=\frac{1}{\Gamma(n-\alpha)} \int_{1}^{t}\left(\log \frac{t}{s}\right)^{n-\alpha-1} \delta^{n} g(s) \frac{d s}{s}, \\
n-1<\alpha<n, \quad n=[\alpha]+1,
\end{array}
$$

where $\delta=t(d / d t), \log (\cdot)=\log _{e}(\cdot)$, and $[\alpha]$ denotes the integer part of the real number $\alpha$.

Definition 2. The Hadamard fractional integral of order $\alpha$ is defined as

$$
I^{\alpha} g(t)=\frac{1}{\Gamma(\alpha)} \int_{1}^{t}\left(\log \frac{t}{s}\right)^{\alpha-1} g(s) \frac{d s}{s}, \quad \alpha>0,
$$

provided that the integral exists.

Lemma 3 (see [35]). Let $u \in A C_{\delta}^{n}[a, b]$ or $C_{\delta}^{n}[a, b]$ and $\alpha \in \mathbb{C}$, where $X_{\delta}^{n}[a, b]=\left\{g:[a, b] \rightarrow \mathbb{C}: \delta^{n-1} g(t) \in X[a, b]\right\}$. Then, one has

$$
I^{\alpha}\left(D^{\alpha}\right) u(t)=u(t)-\sum_{k=0}^{n-1} c_{k}(\log t)^{k},
$$

where $c_{i} \in \mathbb{R}, i=1,2, \ldots, n-1,(n=[\alpha]+1)$.

For convenience, we set constants as follows:

$$
\begin{aligned}
& \Omega_{1}=\sum_{i=1}^{m} \theta_{i} \frac{\left(\log \eta_{i}\right)^{\mu_{i}}}{\Gamma\left(\mu_{i}+1\right)}-\sum_{j=1}^{n} \phi_{j} \frac{\left(\log \omega_{j}\right)^{\gamma_{j}}}{\Gamma\left(\gamma_{j}+1\right)}, \\
& \Omega_{2}=\sum_{i=1}^{m} \theta_{i} \frac{\left(\log \eta_{i}\right)^{\beta+\mu_{i}}}{\Gamma\left(\beta+\mu_{i}+1\right)}-\sum_{j=1}^{n} \phi_{j} \frac{\left(\log \omega_{j}\right)^{\beta+\gamma_{j}}}{\Gamma\left(\beta+\gamma_{j}+1\right)}, \\
& \Omega_{3}=\sum_{k=1}^{p} \varepsilon_{k} \frac{\left(\log \psi_{k}\right)^{\sigma_{k}}}{\Gamma\left(\sigma_{k}+1\right)}-\sum_{l=1}^{q} \nu_{l} \frac{\left(\log \varphi_{l}\right)^{\tau_{l}}}{\Gamma\left(\tau_{l}+1\right)}, \\
& \Omega_{4}=\sum_{k=1}^{p} \varepsilon_{k} \frac{\left(\log \psi_{k}\right)^{\beta+\sigma_{k}}}{\Gamma\left(\beta+\sigma_{k}+1\right)}-\sum_{l=1}^{q} \nu_{l} \frac{\left(\log \varphi_{l}\right)^{\beta+\tau_{l}}}{\Gamma\left(\beta+\tau_{l}+1\right)}, \\
& \Omega=\Omega_{1} \Omega_{4}-\Omega_{2} \Omega_{3} .
\end{aligned}
$$

Lemma 4. Let $\Omega \neq 0,0<\alpha, \beta \leq 1$, and $1<\alpha+\beta \leq 2$; let $\lambda$ be a given constant, $\mu_{i}, \gamma_{j}, \sigma_{k}, \tau_{l}>0$, constants $\eta_{i}, \omega_{j}, \psi_{k}, \varphi_{l} \in$ $(1, e)$, and $\theta_{i}, \phi_{j}, \varepsilon_{k}, v_{l} \in \mathbb{R}$, for $i=1,2, \ldots, m, j=1,2, \ldots, n$, $k=1,2, \ldots, p, l=1,2, \ldots, q$, and $h \in C([1, e], \mathbb{R})$. Then, the problem

$$
\begin{gathered}
D^{\alpha}\left(D^{\beta}+\lambda\right) x(t)=h(t), \quad t \in(1, e), \\
\sum_{i=1}^{m} \theta_{i} I^{\mu_{i}} x\left(\eta_{i}\right)=\sum_{j=1}^{n} \phi_{j} I^{\gamma_{j}} x\left(\omega_{j}\right), \\
\sum_{k=1}^{p} \varepsilon_{k} I^{\sigma_{k}} x\left(\psi_{k}\right)=\sum_{l=1}^{q} \nu_{l} I^{\tau_{l}} x\left(\varphi_{l}\right)
\end{gathered}
$$


has a unique solution given by

$$
\begin{aligned}
x(t)=\frac{1}{\Omega}[ & \left(\Omega_{4}-\frac{(\log t)^{\beta}}{\Gamma(\beta+1)} \Omega_{3}\right) \\
& \times\left(\sum_{j=1}^{n} \phi_{j}\left[I^{\alpha+\beta+\gamma_{j}} h\left(\omega_{j}\right)-\lambda I^{\beta+\gamma_{j}} x\left(\omega_{j}\right)\right]\right. \\
& +\left(\frac{(\log t)^{\beta}}{\Gamma(\beta+1)} \Omega_{1}-\Omega_{2}\right) \\
& \times\left(\sum_{l=1}^{m} \theta_{i}\left[I^{\alpha+\beta+\mu_{i}} h\left(\eta_{i}\right)-\lambda I^{\beta+\mu_{i}} x\left(\eta_{i}\right)\right]\right) \\
& \\
+I^{\alpha+\beta} h(t)-\lambda I^{\beta} x(t) . & \left.\left.-\sum_{k=1}^{p} \varepsilon_{k}\left[I^{\alpha+\beta+\sigma_{k}} h\left(\psi_{k}\right)-\lambda I^{\beta+\sigma_{k}} x\left(\psi_{k}\right)\right]\right)\right]
\end{aligned}
$$

Proof. Using Lemma 3, (7) can be expressed as an equivalent integral equation:

$$
\left(D^{\beta}+\lambda\right) x(t)=I^{\alpha} h(t)+c_{0}
$$

It follows that

$$
x(t)=I^{\alpha+\beta} h(t)-\lambda I^{\beta} x(t)+c_{0} \frac{(\log t)^{\beta}}{\Gamma(\beta+1)}+c_{1},
$$

for some $c_{0}, c_{1} \in \mathbb{R}$.

Taking the Hadamard fractional integral of order $\kappa>0$ for (11), we have

$$
\begin{aligned}
I^{\kappa} x(t)= & I^{\alpha+\beta+\kappa} h(t)-\lambda I^{\beta+\kappa} x(t)+c_{0} \frac{(\log t)^{\beta+\kappa}}{\Gamma(\beta+\kappa+1)} \\
& +c_{1} \frac{(\log t)^{\kappa}}{\Gamma(\kappa+1)} .
\end{aligned}
$$

Substituting $\kappa=\mu_{i}, \gamma_{j}, \sigma_{k}, \tau_{l}$ and putting $t=\eta_{i}, \omega_{j}, \psi_{k}, \varphi_{l}$ in (12), respectively, and using conditions (8), we get the system of linear equations:

$$
\begin{aligned}
\Omega_{1} c_{1}+\Omega_{2} c_{0}= & \sum_{j=1}^{n} \phi_{j}\left[I^{\alpha+\beta+\gamma_{j}} h\left(\omega_{j}\right)-\lambda I^{\beta+\gamma_{j}} x\left(\omega_{j}\right)\right] \\
& -\sum_{i=1}^{m} \theta_{i}\left[I^{\alpha+\beta+\mu_{i}} h\left(\eta_{i}\right)-\lambda I^{\beta+\mu_{i}} x\left(\eta_{i}\right)\right],
\end{aligned}
$$

$$
\begin{aligned}
\Omega_{3} c_{1}+\Omega_{4} c_{0}= & \sum_{l=1}^{p} \nu_{l}\left[I^{\alpha+\beta+\tau_{l}} h\left(\varphi_{l}\right)-\lambda I^{\beta+\tau_{l}} x\left(\varphi_{l}\right)\right] \\
& -\sum_{k=1}^{p} \varepsilon_{k}\left[I^{\alpha+\beta+\sigma_{k}} h\left(\psi_{k}\right)-\lambda I^{\beta+\sigma_{k}} x\left(\psi_{k}\right)\right] .
\end{aligned}
$$

Solving the system of linear equations for constants $c_{0}, c_{1}$, we have

$$
\begin{aligned}
& c_{0}=\frac{1}{\Omega}\left[\Omega _ { 1 } \left(\sum_{l=1}^{p} \nu_{l}\left[I^{\alpha+\beta+\tau_{l}} h\left(\varphi_{l}\right)-\lambda I^{\beta+\tau_{l}} x\left(\varphi_{l}\right)\right]\right.\right. \\
& \left.-\sum_{k=1}^{p} \varepsilon_{k}\left[I^{\alpha+\beta+\sigma_{k}} h\left(\psi_{k}\right)-\lambda I^{\beta+\sigma_{k}} x\left(\psi_{k}\right)\right]\right) \\
& -\Omega_{3}\left(\sum_{j=1}^{n} \phi_{j}\left[I^{\alpha+\beta+\gamma_{j}} h\left(\omega_{j}\right)-\lambda I^{\beta+\gamma_{j}} x\left(\omega_{j}\right)\right]\right. \\
& \left.\left.-\sum_{i=1}^{m} \theta_{i}\left[I^{\alpha+\beta+\mu_{i}} h\left(\eta_{i}\right)-\lambda I^{\beta+\mu_{i}} x\left(\eta_{i}\right)\right]\right)\right], \\
& c_{1}=\frac{1}{\Omega}\left[\Omega _ { 4 } \left(\sum_{j=1}^{n} \phi_{j}\left[I^{\alpha+\beta+\gamma_{j}} h\left(\omega_{j}\right)-\lambda I^{\beta+\gamma_{j}} x\left(\omega_{j}\right)\right]\right.\right. \\
& \left.-\sum_{i=1}^{m} \theta_{i}\left[I^{\alpha+\beta+\mu_{i}} h\left(\eta_{i}\right)-\lambda I^{\beta+\mu_{i}} x\left(\eta_{i}\right)\right]\right) \\
& -\Omega_{2}\left(\sum_{l=1}^{p} \nu_{l}\left[I^{\alpha+\beta+\tau_{l}} h\left(\varphi_{l}\right)-\lambda I^{\beta+\tau_{l}} x\left(\varphi_{l}\right)\right]\right. \\
& -\sum_{k=1}^{p} \varepsilon_{k}\left[I^{\alpha+\beta+\sigma_{k}} h\left(\psi_{k}\right)\right. \\
& \left.\left.\left.-\lambda I^{\beta+\sigma_{k}} x\left(\psi_{k}\right)\right]\right)\right] .
\end{aligned}
$$

Substituting constants $c_{0}$ and $c_{1}$ into (11), we obtain (9) as required.

\section{Main Results}

Let $\mathscr{C}=C([1, e], \mathbb{R})$ denote the Banach space of all continuous functions from $[1, e]$ to $\mathbb{R}$ endowed with the norm defined by $\|x\|=\sup _{t \in[1, e]}|x(t)|$. Throughout this paper, for convenience, the expressions $I^{z} f(s, x(s))(y)$ and $I^{z} x(s)(y)$ mean

$$
\begin{gathered}
I^{z} f(s, x(s))(y)=\frac{1}{\Gamma(z)} \int_{1}^{y}\left(\log \frac{y}{s}\right)^{z-1} \frac{f(s, x(s))}{s} d s \\
I^{z} x(s)(y)=\frac{1}{\Gamma(z)} \int_{1}^{y}\left(\log \frac{y}{s}\right)^{z-1} \frac{x(s)}{s} d s
\end{gathered}
$$

respectively, where $z>0$ and $y \in\left\{t, \eta_{i}, \omega_{j}, \psi_{k}, \varphi_{l}\right\}$. 

$\mathscr{C}$ by

In view of Lemma 4 , we consider the operator $\mathbb{Q}: \mathscr{C} \rightarrow$

$(Q x)(t)$

$$
\begin{gathered}
=\frac{1}{\Omega}\left[\left(\Omega_{4}-\frac{(\log t)^{\beta}}{\Gamma(\beta+1)} \Omega_{3}\right)\right. \\
\times\left(\sum _ { j = 1 } ^ { n } \phi _ { j } \left[I^{\alpha+\beta+\gamma_{j}} f(s, x(s))\left(\omega_{j}\right)\right.\right. \\
\left.-\lambda I^{\beta+\gamma_{j}} x(s)\left(\omega_{j}\right)\right] \\
+\sum_{i=1}^{m} \theta_{i}\left[I^{\alpha+\beta+\mu_{i}} f(s, x(s))\left(\eta_{i}\right)\right. \\
+\left(\frac{(\log t)^{\beta}}{\Gamma(\beta+1)} \Omega_{1}-\Omega_{2}\right) \\
\times\left(\sum_{l=1}^{q} \nu_{l}\left[I^{\alpha+\beta+\mu_{i}} x(s)\left(\eta_{i}\right)\right]\right) \\
-\sum_{k=1}^{p} \varepsilon_{k}\left[I^{\alpha+\beta+\sigma_{k}} f(s, x(s))\left(\psi_{k}\right)\right. \\
\left.\left.\left.-\lambda I^{\beta+\sigma_{k}} x(s)\left(\psi_{k}\right)\right]\right)\left(\varphi_{l}\right)-\lambda I^{\beta+\tau_{l}} x(s)\left(\varphi_{l}\right)\right]
\end{gathered}
$$

It should be noticed that the nonlocal problem (1) has solutions if and only if the operator $Q$ has fixed points.

In the following subsections, we prove existence, as well as existence and uniqueness results, for the nonlocal problem (1) by using a variety of fixed point theorems.

We set

$$
\begin{aligned}
\Lambda(u)=\frac{1}{|\Omega|}\left[\left(\left|\Omega_{4}\right|+\frac{\left|\Omega_{3}\right|}{\Gamma(\beta+1)}\right)\right. \\
\quad \times\left(\sum_{j=1}^{n}\left|\phi_{j}\right| \frac{\left(\log \omega_{j}\right)^{u+\beta+\gamma_{j}}}{\Gamma\left(u+\beta+\gamma_{j}+1\right)}\right. \\
\left.+\sum_{i=1}^{m}\left|\theta_{i}\right| \frac{\left(\log \eta_{i}\right)^{u+\beta+\mu_{i}}}{\Gamma\left(u+\beta+\mu_{i}+1\right)}\right) \\
+\left(\frac{\left|\Omega_{1}\right|}{\Gamma(\beta+1)}+\left|\Omega_{2}\right|\right)
\end{aligned}
$$

$$
\begin{gathered}
\times\left(\sum_{l=1}^{q}\left|\nu_{l}\right| \frac{\left(\log \varphi_{l}\right)^{u+\beta+\tau_{l}}}{\Gamma\left(u+\beta+\tau_{l}+1\right)}\right. \\
\left.\left.\quad+\sum_{k=1}^{p}\left|\varepsilon_{k}\right| \frac{\left(\log \psi_{k}\right)^{u+\beta+\sigma_{k}}}{\Gamma\left(u+\beta+\sigma_{k}+1\right)}\right)\right] \\
+\frac{1}{\Gamma(u+\beta+1)},
\end{gathered}
$$

where $u \in\{0, \alpha\}$.

\subsection{Existence and Uniqueness Result via Banach's Fixed Point Theorem}

Theorem 5. Let $f:[1, e] \times \mathbb{R} \rightarrow \mathbb{R}$ be a continuous function. Assume the following:

$\left(H_{1}\right)$ there exists a constant $L>0$ such that $\mid f(t, x)-$ $f(t, y)|\leq L| x-y \mid$, for each $t \in[1, e]$ and $x, y \in \mathbb{R}$.

If

$$
L \Lambda(\alpha)+|\lambda| \Lambda(0)<1
$$

where constants $\Lambda(0)$ and $\Lambda(\alpha)$ are defined by (18) with $u=0$ and $\alpha$, respectively, then the nonlocal problem (1) has a unique solution on $[1, e]$.

Proof. By transforming the nonlocal problem (1) into a fixed point problem, $x=Q \mathbb{Q} x$, where the operator $Q$ is defined by (17), we have that the fixed points of the operator $Q$ are solutions of problem (1). Applying the Banach contraction mapping principle, we will show that $\mathbb{Q}$ has a unique fixed point.

Setting $\sup _{t \in[1, e]}|f(t, 0)|=M<\infty$ and choosing

$$
R \geq \frac{M \Lambda(\alpha)}{1-L \Lambda(\alpha)-|\lambda| \Lambda(0)},
$$

we show that $Q B_{R} \subset B_{R}$, where $B_{R}=\{x \in \mathscr{C}:\|x\| \leq R\}$. For any $x \in B_{R}$, we have

$$
\begin{aligned}
& |(Q \mathcal{Q} x)(t)| \\
& \leq \mid \frac{1}{\Omega}\left[\left(\Omega_{4}-\frac{(\log t)^{\beta}}{\Gamma(\beta+1)} \Omega_{3}\right)\right. \\
& \quad \times\left(\sum_{j=1}^{n} \phi_{j}\left[I^{\alpha+\beta+\gamma_{j}} f(s, x(s))\left(\omega_{j}\right)-\lambda I^{\beta+\gamma_{j}} x(s)\left(\omega_{j}\right)\right]\right. \\
& -\sum_{i=1}^{m} \theta_{i}\left[I^{\alpha+\beta+\mu_{i}} f(s, x(s))\left(\eta_{i}\right)\right. \\
& \left.\left.-\lambda I^{\beta+\mu_{i}} x(s)\left(\eta_{i}\right)\right]\right)
\end{aligned}
$$




$$
\begin{aligned}
& +\left(\frac{(\log t)^{\beta}}{\Gamma(\beta+1)} \Omega_{1}-\Omega_{2}\right) \\
& \times\left(\sum_{l=1}^{q} v_{l}\left[I^{\alpha+\beta+\tau_{l}} f(s, x(s))\left(\varphi_{l}\right)-\lambda I^{\beta+\tau_{l}} x(s)\left(\varphi_{l}\right)\right]\right. \\
& -\sum_{k=1}^{p} \varepsilon_{k}\left[I^{\alpha+\beta+\sigma_{k}} f(s, x(s))\left(\psi_{k}\right)\right. \\
& \left.\left.\left.-\lambda I^{\beta+\sigma_{k}} x(s)\left(\psi_{k}\right)\right]\right)\right] \\
& +I^{\alpha+\beta} f(s, x(s))(t)-\lambda I^{\beta} x(s)(t) \\
& \leq \frac{1}{|\Omega|}\left[\left|\Omega_{4}-\frac{(\log t)^{\beta}}{\Gamma(\beta+1)} \Omega_{3}\right|\right. \\
& \times\left(\sum _ { j = 1 } ^ { n } | \phi _ { j } | \left[I^{\alpha+\beta+\gamma_{j}}(|f(s, x(s))-f(s, 0)|\right.\right. \\
& +|f(s, 0)|)\left(\omega_{j}\right) \\
& \left.+|\lambda| I^{\beta+\gamma_{j}}|x(s)|\left(\omega_{j}\right)\right] \\
& +\sum_{i=1}^{m}\left|\theta_{i}\right|\left[I^{\alpha+\beta+\mu_{i}}(|f(s, x(s))-f(s, 0)|\right. \\
& +|f(s, 0)|)\left(\eta_{i}\right) \\
& \left.\left.+|\lambda| I^{\beta+\mu_{i}}|x(s)|\left(\eta_{i}\right)\right]\right) \\
& +\left|\frac{(\log t)^{\beta}}{\Gamma(\beta+1)} \Omega_{1}-\Omega_{2}\right| \\
& \times\left(\sum _ { l = 1 } ^ { q } | v _ { l } | \left[I^{\alpha+\beta+\tau_{l}}(|f(s, x(s))-f(s, 0)|\right.\right. \\
& +|f(s, 0)|)\left(\varphi_{l}\right) \\
& \left.+|\lambda| I^{\beta+\tau_{l}}|x(s)|\left(\varphi_{l}\right)\right] \\
& +\sum_{k=1}^{p}\left|\varepsilon_{k}\right|\left[I^{\alpha+\beta+\sigma_{k}}(|f(s, x(s))-f(s, 0)|\right. \\
& +|f(s, 0)|)\left(\psi_{k}\right) \\
& \left.\left.\left.+|\lambda| I^{\beta+\sigma_{k}}|x(s)|\left(\psi_{k}\right)\right]\right)\right] \\
& +I^{\alpha+\beta}(|f(s, x(s))-f(s, 0)|+|f(s, 0)|)(t) \\
& +|\lambda| I^{\beta}|x(s)|(t)
\end{aligned}
$$$$
\leq \frac{1}{|\Omega|}\left[\left(\left|\Omega_{4}\right|+\frac{(\log t)^{\beta}}{\Gamma(\beta+1)}\left|\Omega_{3}\right|\right)\right.
$$$$
\begin{gathered}
\times\left(\sum _ { j = 1 } ^ { n } | \phi _ { j } | \left[(L\|x\|+M)\left(I^{\alpha+\beta+\gamma_{j}} 1\right)\left(\omega_{j}\right)\right.\right. \\
\left.+|\lambda|\|x\|\left(I^{\beta+\gamma_{j}} 1\right)\left(\omega_{j}\right)\right] \\
+\sum_{i=1}^{m}\left|\theta_{i}\right|\left[(L\|x\|+M)\left(I^{\alpha+\beta+\mu_{i}} 1\right)\left(\eta_{i}\right)\right.
\end{gathered}
$$$$
\left.\left.+|\lambda|\|x\|\left(I^{\beta+\mu_{i}} 1\right)\left(\eta_{i}\right)\right]\right)
$$$$
+\left(\frac{(\log t)^{\beta}}{\Gamma(\beta+1)}\left|\Omega_{1}\right|+\left|\Omega_{2}\right|\right)
$$$$
\times\left(\sum _ { l = 1 } ^ { q } | \nu _ { l } | \left[(L\|x\|+M)\left(I^{\alpha+\beta+\tau_{l}} 1\right)\left(\varphi_{l}\right)\right.\right.
$$$$
\left.+|\lambda|\|x\|\left(I^{\beta+\tau_{l}} 1\right)\left(\varphi_{l}\right)\right]
$$$$
+\sum_{k=1}^{p}\left|\varepsilon_{k}\right|\left[(L\|x\|+M)\left(I^{\alpha+\beta+\sigma_{k}} 1\right)\left(\psi_{k}\right)\right.
$$$$
\left.\left.\left.+|\lambda|\|x\|\left(I^{\beta+\sigma_{k}} 1\right)\left(\psi_{k}\right)\right]\right)\right]
$$$$
+(L\|x\|+M)\left(I^{\alpha+\beta} 1\right)(t)+|\lambda|\|x(s)\|\left(I^{\beta} 1\right)(t)
$$$$
\leq \frac{1}{|\Omega|}\left[\left(\left|\Omega_{4}\right|+\frac{(\log t)^{\beta}}{\Gamma(\beta+1)}\left|\Omega_{3}\right|\right)\right.
$$$$
\times\left(\sum _ { j = 1 } ^ { n } | \phi _ { j } | \left[(L R+M) \frac{\left(\log \omega_{j}\right)^{\alpha+\beta+\gamma_{j}}}{\Gamma\left(\alpha+\beta+\gamma_{j}+1\right)}\right.\right.
$$$$
\left.+|\lambda| R \frac{\left(\log \omega_{j}\right)^{\beta+\gamma_{j}}}{\Gamma\left(\beta+\gamma_{j}+1\right)}\right]
$$$$
+\sum_{i=1}^{m}\left|\theta_{i}\right|\left[(L R+M) \frac{\left(\log \eta_{i}\right)^{\alpha+\beta+\mu_{i}}}{\Gamma\left(\alpha+\beta+\mu_{i}+1\right)}\right.
$$$$
\left.\left.+|\lambda| R \frac{\left(\log \eta_{i}\right)^{\beta+\mu_{i}}}{\Gamma\left(\beta+\mu_{i}+1\right)}\right]\right)
$$$$
+\left(\frac{(\log t)^{\beta}}{\Gamma(\beta+1)}\left|\Omega_{1}\right|+\left|\Omega_{2}\right|\right)
$$$$
\times\left(\sum _ { l = 1 } ^ { q } | v _ { l } | \left[(L R+M) \frac{\left(\log \varphi_{l}\right)^{\alpha+\beta+\tau_{l}}}{\Gamma\left(\alpha+\beta+\tau_{l}+1\right)}\right.\right.
$$$$
\left.+|\lambda| R \frac{\left(\log \varphi_{l}\right)^{\beta+\tau_{l}}}{\Gamma\left(\beta+\tau_{l}+1\right)}\right]
$$ 


$$
\begin{array}{r}
+\sum_{k=1}^{p}\left|\varepsilon_{k}\right|\left[(L R+M) \frac{\left(\log \psi_{k}\right)^{\alpha+\beta+\sigma_{k}}}{\Gamma\left(\alpha+\beta+\sigma_{k}+1\right)}\right. \\
\left.\left.\left.+|\lambda| R \frac{\left(\log \psi_{k}\right)^{\beta+\sigma_{k}}}{\Gamma\left(\beta+\sigma_{k}+1\right)}\right]\right)\right] \\
+(L R+M) \frac{(\log t)^{\alpha+\beta}}{\Gamma(\alpha+\beta+1)}+|\lambda| R \frac{(\log t)^{\beta}}{\Gamma(\beta+1)} \\
\leq(L R+M) \Lambda(\alpha)+|\lambda| R \Lambda(0) \\
=[L \Lambda(\alpha)+|\lambda| \Lambda(0)] R+M \Lambda(\alpha) \leq R .
\end{array}
$$

This implies that $\|\mathbb{Q} x\| \leq R$ for $x \in B_{R}$. Therefore, $\mathbb{Q}$ maps bounded subsets of $B_{R}$ into bounded subsets of $B_{R}$.

Next, we let $x, y \in \mathscr{C}$. Then, for $t \in[1, e]$, we have

$$
\begin{aligned}
& |(\mathscr{Q} x)(t)-(\mathscr{Q} y)(t)| \\
& \leq \frac{1}{|\Omega|}\left[\left|\Omega_{4}-\frac{(\log t)^{\beta}}{\Gamma(\beta+1)} \Omega_{3}\right|\right. \\
& \times\left(\sum _ { j = 1 } ^ { n } | \phi _ { j } | \left[I^{\alpha+\beta+\gamma_{j}}(|f(s, x(s))-f(s, y(s))|)\left(\omega_{j}\right)\right.\right. \\
& \left.+|\lambda| I^{\beta+\gamma_{j}}(|x(s)-y(s)|)\left(\omega_{j}\right)\right] \\
& +\sum_{i=1}^{m}\left|\theta_{i}\right|\left[I^{\alpha+\beta+\mu_{i}}\right. \\
& \times(|f(s, x(s))-f(s, y(s))|)\left(\eta_{i}\right) \\
& \left.\left.+|\lambda| I^{\beta+\mu_{i}}(|x(s)-y(s)|)\left(\eta_{i}\right)\right]\right) \\
& +\left|\frac{(\log t)^{\beta}}{\Gamma(\beta+1)} \Omega_{1}-\Omega_{2}\right| \\
& \times\left(\sum _ { l = 1 } ^ { q } | \nu _ { l } | \left[I^{\alpha+\beta+\tau_{l}}(|f(s, x(s))-f(s, y(s))|)\left(\varphi_{l}\right)\right.\right. \\
& \left.+|\lambda| I^{\beta+\tau_{l}}(|x(s)-y(s)|)\left(\varphi_{l}\right)\right] \\
& +\sum_{k=1}^{p}\left|\varepsilon_{k}\right|\left[I^{\alpha+\beta+\sigma_{k}}\right. \\
& \times(|f(s, x(s))-f(s, y(s))|) \\
& \times\left(\psi_{k}\right)+|\lambda| I^{\beta+\sigma_{k}} \\
& \left.\left.\left.\times(|x(s)-y(s)|)\left(\psi_{k}\right)\right]\right)\right]
\end{aligned}
$$

$$
\begin{aligned}
& +I^{\alpha+\beta}(|f(s, x(s))-f(s, y(s))|)(t) \\
& +|\lambda| I^{\beta}(|x(s)-y(s)|)(t) \\
& \leq \frac{1}{|\Omega|}\left[\left(\left|\Omega_{4}\right|+\frac{(\log t)^{\beta}}{\Gamma(\beta+1)}\left|\Omega_{3}\right|\right)\right. \\
& \times\left(\sum _ { j = 1 } ^ { n } | \phi _ { j } | \left[(L\|x-y\|) \frac{\left(\log \omega_{j}\right)^{\alpha+\beta+\gamma_{j}}}{\Gamma\left(\alpha+\beta+\gamma_{j}+1\right)}\right.\right. \\
& \left.+|\lambda|\|x-y\| \frac{\left(\log \omega_{j}\right)^{\beta+\gamma_{j}}}{\Gamma\left(\beta+\gamma_{j}+1\right)}\right] \\
& +\sum_{i=1}^{m}\left|\theta_{i}\right|\left[(L\|x-y\|) \frac{\left(\log \eta_{i}\right)^{\alpha+\beta+\mu_{i}}}{\Gamma\left(\alpha+\beta+\mu_{i}+1\right)}\right. \\
& \left.\left.+|\lambda|\|x-y\| \frac{\left(\log \eta_{i}\right)^{\beta+\mu_{i}}}{\Gamma\left(\beta+\mu_{i}+1\right)}\right]\right) \\
& +\left(\frac{(\log t)^{\beta}}{\Gamma(\beta+1)}\left|\Omega_{1}\right|+\left|\Omega_{2}\right|\right) \\
& \times\left(\sum _ { l = 1 } ^ { q } | v _ { l } | \left[(L\|x-y\|) \frac{\left(\log \varphi_{l}\right)^{\alpha+\beta+\tau_{l}}}{\Gamma\left(\alpha+\beta+\tau_{l}+1\right)}\right.\right. \\
& \left.+|\lambda|\|x-y\| \frac{\left(\log \varphi_{l}\right)^{\beta+\tau_{l}}}{\Gamma\left(\beta+\tau_{l}+1\right)}\right] \\
& +\sum_{k=1}^{p}\left|\varepsilon_{k}\right|\left[(L\|x-y\|) \frac{\left(\log \psi_{k}\right)^{\alpha+\beta+\sigma_{k}}}{\Gamma\left(\alpha+\beta+\sigma_{k}+1\right)}\right. \\
& \left.\left.\left.+|\lambda|\|x-y\| \frac{\left(\log \psi_{k}\right)^{\beta+\sigma_{k}}}{\Gamma\left(\beta+\sigma_{k}+1\right)}\right]\right)\right] \\
& +(L\|x-y\|) \frac{(\log t)^{\alpha+\beta}}{\Gamma(\alpha+\beta+1)}+|\lambda|\|x-y\| \frac{(\log t)^{\beta}}{\Gamma(\beta+1)} \\
& \leq L \Lambda(\alpha)\|x-y\|+|\lambda|\|x-y\| \Lambda(0) \\
& =[L \Lambda(\alpha)+|\lambda| \Lambda(0)]\|x-y\|,
\end{aligned}
$$

which implies that $\|\mathscr{Q} x-\mathscr{Q} y\| \leq[L \Lambda(\alpha)+|\lambda| \Lambda(0)]\|x-y\|$. As $[L \Lambda(\alpha)+|\lambda| \Lambda(0)]<1, \mathbb{Q}$ is a contraction. Therefore, we deduce, by the Banach contraction mapping principle, that $Q$ has a fixed point which is the unique solution of nonlocal problem (1). The proof is completed.

\subsection{Existence Result via Krasnoselskii's Fixed Point Theorem}

Lemma 6 (Krasnoselskii's fixed point theorem) (see [36]). Let $M$ be a closed, bounded, convex, and nonempty subset of a Banach space X. Let $A, B$ be the operators such that (a) Ax+ $B y \in M$ whenever $x, y \in M$; (b) $A$ is compact and continuous; 
(c) $B$ is a contraction mapping. Then, there exists $z \in M$ such that $z=A z+B z$.

Theorem 7. Let $f:[1, e] \times \mathbb{R} \rightarrow \mathbb{R}$ be a continuous function. Assume the following:

$\left(H_{2}\right)|f(t, x)| \leq \rho(t), \forall(t, x) \in[1, e] \times \mathbb{R}$ and $\rho \in$ $C\left([1, e], \mathbb{R}^{+}\right)$.

If

$$
|\lambda| \Lambda(0)<1
$$

where $\Lambda(0)$ is defined by (18), with $u=0$, then the nonlocal problem (1) has at least one solution on $[1, e]$.

Proof. Setting $\sup _{t \in[1, e]}|\rho(t)|=\|\rho\|$ and choosing

$$
R \geq \frac{\|\rho\| \Lambda(\alpha)}{1-\|\lambda\| \Lambda(0)},
$$

we consider $B_{R}=\{x \in \mathscr{C}([1, e], \mathbb{R}):\|x\| \leq R\}$. We define the operators $\mathbb{Q}_{1}$ and $\mathcal{Q}_{2}$ on $B_{R}$ by

$$
\begin{aligned}
& \left(Q_{1} x\right)(t)=\frac{1}{\Omega}\left[\left(\Omega_{4}-\frac{(\log t)^{\beta}}{\Gamma(\beta+1)} \Omega_{3}\right)\right. \\
& \times\left(\sum_{j=1}^{n} \phi_{j}\left[I^{\alpha+\beta+\gamma_{j}} f(s, x(s))\left(\omega_{j}\right)\right]\right. \\
& \left.-\sum_{i=1}^{m} \theta_{i}\left[I^{\alpha+\beta+\mu_{i}} f(s, x(s))\left(\eta_{i}\right)\right]\right) \\
& +\left(\frac{(\log t)^{\beta}}{\Gamma(\beta+1)} \Omega_{1}-\Omega_{2}\right) \\
& \times\left(\sum_{l=1}^{q} v_{l}\left[I^{\alpha+\beta+\tau_{l}} f(s, x(s))\left(\varphi_{l}\right)\right]\right. \\
& \left.\left.-\sum_{k=1}^{p} \varepsilon_{k}\left[I^{\alpha+\beta+\sigma_{k}} f(s, x(s))\left(\psi_{k}\right)\right]\right)\right] \\
& +I^{\alpha+\beta} f(s, x(s))(t), \\
& \left(Q_{2} x\right)(t)=\frac{1}{\Omega}\left[\left(\Omega_{4}-\frac{(\log t)^{\beta}}{\Gamma(\beta+1)} \Omega_{3}\right)\right. \\
& \times\left(\sum_{j=1}^{n}-\phi_{j}\left[\lambda I^{\beta+\gamma_{j}} x(s)\left(\omega_{j}\right)\right]\right. \\
& \left.+\sum_{i=1}^{m} \theta_{i}\left[\lambda I^{\beta+\mu_{i}} x(s)\left(\eta_{i}\right)\right]\right) \\
& +\left(\frac{(\log t)^{\beta}}{\Gamma(\beta+1)} \Omega_{1}-\Omega_{2}\right)
\end{aligned}
$$

$$
\begin{gathered}
\times\left(\sum_{l=1}^{q}-v_{l}\left[\lambda I^{\beta+\tau_{l}} x(s)\left(\varphi_{l}\right)\right]\right. \\
\left.\left.+\sum_{k=1}^{p} \varepsilon_{k}\left[\lambda I^{\beta+\sigma_{k}} x(s)\left(\psi_{k}\right)\right]\right)\right] \\
-\lambda I^{\beta} x(s)(t), \quad t \in[1, e] .
\end{gathered}
$$

For any $x, y \in B_{R}$, we have

$$
\begin{gathered}
\left|Q_{1} x(t)+Q_{2} y(t)\right| \\
\leq \frac{1}{|\Omega|}\left[\left|\Omega_{4}-\frac{(\log t)^{\beta}}{\Gamma(\beta+1)} \Omega_{3}\right|\right. \\
\times\left(\sum _ { j = 1 } ^ { n } | \phi _ { j } | \left[\|\rho\|\left(I^{\alpha+\beta+\gamma_{j}} 1\right)\left(\omega_{j}\right)\right.\right. \\
\left.+|\lambda| R\left(I^{\beta+\gamma_{j}} 1\right)\left(\omega_{j}\right)\right] \\
+\sum_{i=1}^{m}\left|\theta_{i}\right|\left[\|\rho\|\left(I^{\alpha+\beta+\mu_{i}} 1\right)\left(\eta_{i}\right)\right. \\
+\|\rho\| \Lambda(\alpha)+R\|\lambda\| \Lambda(0) \leq R, \\
\left.\left.+\left|\frac{(\log t)^{\beta}}{\Gamma(\beta+1)} \Omega_{1}-\Omega_{2}\right| \lambda\left(I^{\beta+\mu_{i}} 1\right)\left(\eta_{i}\right)\right]\right) \\
+\left(\sum _ { l = 1 } ^ { q } | \nu _ { l } | \left[\|\rho\|\left(I^{\alpha+\beta+\tau_{l}} 1\right)\left(\varphi_{l}\right)\right.\right. \\
+\sum_{k=1}^{p}\left|\varepsilon_{k}\right|\left[\|\rho\|\left(I^{\alpha+\beta+\sigma_{k}} 1\right)\left(\psi_{k}\right)\right. \\
\left.+|\lambda| R\left(I^{\beta+\tau_{l}} 1\right)\left(\varphi_{l}\right)\right]
\end{gathered}
$$

which implies that $\left\|Q_{1} x+Q_{2} y\right\| \leq R$. It follows that $\mathbb{Q}_{1} x+$ $Q_{2} y \in B_{R}$.

For $x, y \in C$ and for each $t \in[1, e]$, we have

$$
\left\|Q_{2} x-Q_{2} y\right\| \leq|\lambda| \Lambda(0)\|x-y\| .
$$

Hence, by (23), $Q_{2}$ is a contraction mapping. Continuity of $f$ implies that the operator $\mathbb{Q}_{1}$ is continuous. Also, $\mathbb{Q}_{1}$ is uniformly bounded on $B_{R}$ as

$$
\left\|Q_{1} x\right\| \leq\|\rho\| \Lambda(\alpha) .
$$

Now, we prove the compactness of the operator $\mathbb{Q}_{1}$. 
We define $\sup _{(t, x) \in\left[(1, e) \times B_{R}\right]}|f(t, x)|=\bar{f}<\infty$. Let $t_{1}, t_{2} \in$ $[1, e]$ with $t_{1}<t_{2}$ and $x \in B_{R}$. Then, we have

$$
\begin{aligned}
& \left|\left(Q_{1} x\right)\left(t_{2}\right)-\left(Q_{1} x\right)\left(t_{1}\right)\right| \\
& \leq \mid \frac{1}{\Omega}\left[\Omega_{3}\left(\frac{\left(\log t_{2}\right)^{\beta}-\left(\log t_{1}\right)^{\beta}}{\Gamma(\beta+1)}\right)\right. \\
& \times\left(\sum_{j=1}^{n} \phi_{j}\left[I^{\alpha+\beta+\gamma_{j}} f(s, x(s))\left(\omega_{j}\right)\right]\right. \\
& \left.-\sum_{i=1}^{m} \theta_{i}\left[I^{\alpha+\beta+\mu_{i}} f(s, x(s))\left(\eta_{i}\right)\right]\right) \\
& +\Omega_{1}\left(\frac{\left(\log t_{2}\right)^{\beta}-\left(\log t_{1}\right)^{\beta}}{\Gamma(\beta+1)}\right) \\
& \times\left(\sum_{l=1}^{q} \nu_{l}\left[I^{\alpha+\beta+\tau_{l}} f(s, x(s))\left(\varphi_{l}\right)\right]\right. \\
& \left.\left.-\sum_{k=1}^{p} \varepsilon_{k}\left[I^{\alpha+\beta+\sigma_{k}} f(s, x(s))\left(\psi_{k}\right)\right]\right)\right] \mid \\
& +\left|I^{\alpha+\beta} f(s, x(s))\left(t_{2}\right)-I^{\alpha+\beta} f(s, x(s))\left(t_{1}\right)\right| \\
& \leq \frac{\bar{f}}{|\Omega|}\left[\left|\Omega_{3}\right|\left|\frac{\left(\log t_{1}\right)^{\beta}-\left(\log t_{2}\right)^{\beta}}{\Gamma(\beta+1)}\right|\right. \\
& \times\left(\sum_{j=1}^{n}\left|\phi_{j}\right| \frac{\left(\log \omega_{j}\right)^{\alpha+\beta+\gamma_{j}}}{\Gamma\left(\alpha+\beta+\gamma_{j}+1\right)}\right. \\
& \left.+\sum_{i=1}^{m}\left|\theta_{i}\right| \frac{\left(\log \eta_{i}\right)^{\alpha+\beta+\mu_{i}}}{\Gamma\left(\alpha+\beta+\mu_{i}+1\right)}\right) \\
& +\left|\Omega_{1}\right|\left|\frac{\left(\log t_{2}\right)^{\beta}-\left(\log t_{1}\right)^{\beta}}{\Gamma(\beta+1)}\right| \\
& \times\left(\sum_{l=1}^{q}\left|\nu_{l}\right| \frac{\left(\log \varphi_{l}\right)^{\alpha+\beta+\tau_{l}}}{\Gamma\left(\alpha+\beta+\tau_{l}+1\right)}\right. \\
& \left.\left.+\sum_{k=1}^{p}\left|\varepsilon_{k}\right| \frac{\left(\log \psi_{k}\right)^{\alpha+\beta+\sigma_{k}}}{\Gamma\left(\alpha+\beta+\sigma_{k}+1\right)}\right)\right] \\
& +\frac{\bar{f}}{\Gamma(\alpha+\beta+1)}\left|\left(\log t_{2}\right)^{\alpha+\beta}-\left(\log t_{1}\right)^{\alpha+\beta}\right| \text {, }
\end{aligned}
$$

which is independent of $x$ and tends to zero as $t_{2}-t_{1} \rightarrow 0$. Thus, $Q_{1}$ is equicontinuous. So $\mathbb{Q}_{1}$ is relatively compact on $B_{R}$. Hence, by the Arzelá-Ascoli theorem, $Q_{1}$ is compact on $B_{R}$. Thus, all the assumptions of Lemma 6 are satisfied. So the conclusion of Lemma 6 implies that the nonlocal problem (1) has at least one solution on $[1, e]$.

\subsection{Existence Result via Leray-Schauder's} Nonlinear Alternative

Theorem 8 (nonlinear alternative for single valued maps) (see [37]). Let $E$ be a Banach space; let $C$ be a closed, convex subset of $E$; let $U$ be an open subset of $C$ and $0 \in U$. Suppose that $F: \bar{U} \rightarrow C$ is a continuous, compact (i.e., $F(\bar{U})$ is a relatively compact subset of C) map. Then, either

(i) F has a fixed point in $\bar{U}$, or

(ii) there is a $u \in \partial U$ (the boundary of $U$ in $C$ ) and $\xi \epsilon$ $(0,1)$ with $u=\xi F(u)$.

Theorem 9. Let $f:[1, e] \times \mathbb{R} \rightarrow \mathbb{R}$ be a continuous function. Assume the following:

$\left(\mathrm{H}_{3}\right)$ there exist a continuous nondecreasing function $\Upsilon$ : $[0, \infty) \rightarrow(0, \infty)$ and a function $\rho \in C\left([1, e], \mathbb{R}^{+}\right)$ such that

$|f(t, u)| \leq \rho(t) \Upsilon(\|u\|) \quad$ for each $(t, u) \in[1, e] \times \mathbb{R} ;$

$\left(H_{4}\right)$ there exists a constant $M>0$ such that

$$
\frac{M}{\|\rho\| \Upsilon(M) \Lambda(\alpha)+|\lambda| M \Lambda(0)}>1
$$

where $\Lambda(0)$ and $\Lambda(\alpha)$ are defined by (18) with $u=0$ and $\alpha$, respectively.

Then, the nonlocal problem (1) has at least one solution on $[1, e]$.

Proof. Let the operator $Q$ be defined by (17). Firstly, we will show that $Q$ maps bounded sets (balls) into bounded sets in $C([1, e], \mathbb{R})$. For a number $R>0$, let $B_{R}=\{x \in C([1, e], \mathbb{R})$ : $\|x\| \leq R\}$ be a bounded ball in $C([1, e], \mathbb{R})$. Then, for $t \in[1, e]$, we have

$$
\begin{aligned}
& |(Q \mathcal{Q} x)(t)| \\
& \leq \mid \frac{1}{\Omega}\left[\left(\Omega_{4}-\frac{(\log t)^{\beta}}{\Gamma(\beta+1)} \Omega_{3}\right)\right. \\
& \quad \times\left(\sum_{j=1}^{n} \phi_{j}\left[I^{\alpha+\beta+\gamma_{j}} f(s, x(s))\left(\omega_{j}\right)-\lambda I^{\beta+\gamma_{j}} x(s)\left(\omega_{j}\right)\right]\right. \\
& \quad-\sum_{i=1}^{m} \theta_{i}\left[I^{\alpha+\beta+\mu_{i}} f(s, x(s))\left(\eta_{i}\right)\right. \\
& \left.\left.-\lambda I^{\beta+\mu_{i}} x(s)\left(\eta_{i}\right)\right]\right)
\end{aligned}
$$




$$
\begin{aligned}
& +\left(\frac{(\log t)^{\beta}}{\Gamma(\beta+1)} \Omega_{1}-\Omega_{2}\right) \\
& \times\left(\sum_{l=1}^{q} v_{l}\left[I^{\alpha+\beta+\tau_{l}} f(s, x(s))\left(\varphi_{l}\right)-\lambda I^{\beta+\tau_{l}} x(s)\left(\varphi_{l}\right)\right]\right. \\
& -\sum_{k=1}^{p} \varepsilon_{k}\left[I^{\alpha+\beta+\sigma_{k}} f(s, x(s))\left(\psi_{k}\right)\right. \\
& \left.\left.\left.-\lambda I^{\beta+\sigma_{k}} x(s)\left(\psi_{k}\right)\right]\right)\right] \\
& +I^{\alpha+\beta} f(s, x(s))(t)-\lambda I^{\beta} x(s)(t) \\
& \leq \frac{1}{|\Omega|}\left[\left(\left|\Omega_{4}\right|+\frac{(\log t)^{\beta}}{\Gamma(\beta+1)}\left|\Omega_{3}\right|\right)\right. \\
& \times\left(\sum _ { j = 1 } ^ { n } | \phi _ { j } | \left[I^{\alpha+\beta+\gamma_{j}}\|\rho\| \Upsilon(\|x\|)\left(\omega_{j}\right)\right.\right. \\
& \left.+|\lambda| I^{\beta+\gamma_{j}}\|x\|\left(\omega_{j}\right)\right] \\
& +\sum_{i=1}^{m}\left|\theta_{i}\right|\left[I^{\alpha+\beta+\mu_{i}}\|\rho\| \Upsilon(\|x\|)\left(\eta_{i}\right)\right. \\
& \left.\left.+|\lambda| I^{\beta+\mu_{i}}\|x\|\left(\eta_{i}\right)\right]\right) \\
& +\left(\frac{(\log t)^{\beta}}{\Gamma(\beta+1)}\left|\Omega_{1}\right|+\left|\Omega_{2}\right|\right) \\
& \times\left(\sum _ { l = 1 } ^ { q } | \nu _ { l } | \left[I^{\alpha+\beta+\tau_{l}}\|\rho\| \Upsilon(\|x\|)\left(\varphi_{l}\right)\right.\right. \\
& \left.+|\lambda| I^{\beta+\tau_{l}}\|x\|\left(\varphi_{l}\right)\right] \\
& +\sum_{k=1}^{p}\left|\varepsilon_{k}\right|\left[I^{\alpha+\beta+\sigma_{k}}\|\rho\| \Upsilon(\|x\|)\left(\psi_{k}\right)\right. \\
& \left.\left.\left.+|\lambda| I^{\beta+\sigma_{k}}\|x\|\left(\psi_{k}\right)\right]\right)\right] \\
& +I^{\alpha+\beta}\|\rho\| \Upsilon(\|x\|)(t)+|\lambda| I^{\beta}\|x\|(t) \\
& \leq\|\rho\| \Upsilon(\|x\|) \Lambda(\alpha)+|\lambda| R \Lambda(0) \\
& \leq\|\rho\| \Upsilon(R) \Lambda(\alpha)+|\lambda| R \Lambda(0),
\end{aligned}
$$

and, consequently,

$$
\|Q \mathbb{Q} x\| \leq\|\rho\| \Upsilon(R) \Lambda(\alpha)+|\lambda| R \Lambda(0) .
$$

Next, we will show that $\mathbb{Q}$ maps bounded sets into equicontinuous sets of $C([1, e], \mathbb{R})$. Let $t_{1}, t_{2} \in[1, e]$ with $t_{1}<t_{2}$ and $x \in B_{R}$. Then, we have

$$
\begin{aligned}
& \left|(Q \mathcal{Q} x)\left(t_{2}\right)-(\mathbb{Q} x)\left(t_{1}\right)\right| \\
& \leq \mid \frac{1}{\Omega}\left[\frac{\left(\log t_{2}\right)^{\beta}-\left(\log t_{1}\right)^{\beta}}{\Gamma(\beta+1)} \Omega_{3}\right. \\
& \quad \times\left(\sum _ { j = 1 } ^ { n } \phi _ { j } \left[I^{\alpha+\beta+\gamma_{j}} f(s, x(s))\left(\omega_{j}\right)\right.\right.
\end{aligned}
$$$$
\left.-\lambda I^{\beta+\gamma_{j}} x(s)\left(\omega_{j}\right)\right]
$$$$
-\sum_{i=1}^{m} \theta_{i}\left[I^{\alpha+\beta+\mu_{i}} f(s, x(s))\left(\eta_{i}\right)\right.
$$

$$
\left.\left.-\lambda I^{\beta+\mu_{i}} x(s)\left(\eta_{i}\right)\right]\right)
$$

$$
+\frac{\left(\log t_{2}\right)^{\beta}-\left(\log t_{1}\right)^{\beta}}{\Gamma(\beta+1)} \Omega_{1}
$$$$
\times\left(\sum _ { l = 1 } ^ { q } \nu _ { l } \left[I^{\alpha+\beta+\tau_{l}} f(s, x(s))\left(\varphi_{l}\right)\right.\right.
$$$$
\left.-\lambda I^{\beta+\tau_{l}} x(s)\left(\varphi_{l}\right)\right]
$$$$
-\sum_{k=1}^{p} \varepsilon_{k}\left[I^{\alpha+\beta+\sigma_{k}} f(s, x(s))\left(\psi_{k}\right)\right.
$$

$$
\left.\left.\left.-\lambda I^{\beta+\sigma_{k}} x(s)\left(\psi_{k}\right)\right]\right)\right] \mid
$$

$$
\begin{aligned}
& +\mid I^{\alpha+\beta} f(s, x(s))\left(t_{2}\right)-\lambda I^{\beta} x(s)\left(t_{2}\right) \\
& \quad-I^{\alpha+\beta} f(s, x(s))\left(t_{1}\right)+\lambda I^{\beta} x(s)\left(t_{1}\right) \mid
\end{aligned}
$$$$
\leq \frac{1}{|\Omega|}\left[\left|\frac{\left(\log t_{2}\right)^{\beta}-\left(\log t_{1}\right)^{\beta}}{\Gamma(\beta+1)}\right|\left|\Omega_{3}\right|\right.
$$

$$
\begin{array}{r}
\times\left(\sum _ { j = 1 } ^ { n } | \phi _ { j } | \left[I^{\alpha+\beta+\gamma_{j}}\|\rho\| \Upsilon(\|x\|)\left(\omega_{j}\right)\right.\right. \\
\left.+|\lambda| I^{\beta+\gamma_{j}}\|x\|\left(\omega_{j}\right)\right]
\end{array}
$$

$+\sum_{i=1}^{m}\left|\theta_{i}\right|\left[I^{\alpha+\beta+\mu_{i}}\|\rho\| \Upsilon(\|x\|)\left(\eta_{i}\right)\right.$

$$
\left.\left.+|\lambda| I^{\beta+\mu_{i}}\|x\|\left(\eta_{i}\right)\right]\right)
$$




$$
\begin{aligned}
& +\left|\frac{\left(\log t_{2}\right)^{\beta}-\left(\log t_{1}\right)^{\beta}}{\Gamma(\beta+1)}\right|\left|\Omega_{1}\right| \\
& \times\left(\sum _ { l = 1 } ^ { q } | v _ { l } | \left[I^{\alpha+\beta+\tau_{l}}\|\rho\| \Upsilon(\|x\|)\left(\varphi_{l}\right)\right.\right. \\
& \left.+|\lambda| I^{\beta+\tau_{l}}\|x\|\left(\varphi_{l}\right)\right] \\
& +\sum_{k=1}^{p}\left|\varepsilon_{k}\right|\left[I^{\alpha+\beta+\sigma_{k}}\|\rho\| \Upsilon(\|x\|)\left(\psi_{k}\right)\right. \\
& \left.\left.\left.+|\lambda| I^{\beta+\sigma_{k}}\|x\|\left(\psi_{k}\right)\right]\right)\right] \\
& +\frac{\|\rho\| \Upsilon(\|x\|)}{\Gamma(\alpha+\beta+1)}\left|\left(\log t_{2}\right)^{\alpha+\beta}-\left(\log t_{1}\right)^{\alpha+\beta}\right| \\
& +\frac{|\lambda|\|x\|}{\Gamma(\beta+1)}\left|\left(\log t_{1}\right)^{\beta}-\left(\log t_{2}\right)^{\beta}+2\left(\log \frac{t_{2}}{t_{1}}\right)^{\beta}\right| \\
& \leq \frac{1}{|\Omega|}\left[\left|\frac{\left(\log t_{2}\right)^{\beta}-\left(\log t_{1}\right)^{\beta}}{\Gamma(\beta+1)}\right|\left|\Omega_{3}\right|\right. \\
& \times\left(\sum _ { j = 1 } ^ { n } | \phi _ { j } | \left[\frac{\|\rho\| \Upsilon(R)\left(\log \omega_{j}\right)^{\alpha+\beta+\gamma_{j}}}{\Gamma\left(\alpha+\beta+\gamma_{j}+1\right)}\right.\right. \\
& \left.+\frac{|\lambda| R\left(\log \omega_{j}\right)^{\beta+\gamma_{j}}}{\Gamma\left(\beta+\gamma_{j}+1\right)}\right] \\
& +\sum_{i=1}^{m}\left|\theta_{i}\right|\left[\frac{\|\rho\| \Upsilon(R)\left(\log \eta_{i}\right)^{\alpha+\beta+\mu_{i}}}{\Gamma\left(\alpha+\beta+\mu_{i}+1\right)}\right. \\
& \left.\left.+\frac{|\lambda| R\left(\log \eta_{i}\right)^{\beta+\mu_{i}}}{\Gamma\left(\beta+\mu_{i}+1\right)}\right]\right) \\
& +\left|\frac{\left(\log t_{2}\right)^{\beta}-\left(\log t_{1}\right)^{\beta}}{\Gamma(\beta+1)}\right|\left|\Omega_{1}\right| \\
& \times\left(\sum _ { l = 1 } ^ { q } | \nu _ { l } | \left[\frac{\|\rho\| \Upsilon(R)\left(\log \varphi_{l}\right)^{\alpha+\beta+\tau_{l}}}{\Gamma\left(\alpha+\beta+\tau_{l}+1\right)}\right.\right. \\
& \left.+\frac{|\lambda| R\left(\log \varphi_{l}\right)^{\beta+\tau_{l}}}{\Gamma\left(\beta+\tau_{l}+1\right)}\right] \\
& +\sum_{k=1}^{p}\left|\varepsilon_{k}\right|\left[\frac{\|\rho\| \Upsilon(R)\left(\log \psi_{k}\right)^{\alpha+\beta+\sigma_{k}}}{\Gamma\left(\alpha+\beta+\sigma_{k}+1\right)}\right. \\
& \left.\left.\left.+\frac{|\lambda| R\left(\log \psi_{k}\right)^{\beta+\sigma_{k}}}{\Gamma\left(\beta+\sigma_{k}+1\right)}\right]\right)\right]
\end{aligned}
$$

As $t_{2}-t_{1} \rightarrow 0$, the right-hand side of the above inequality tends to zero independently of $x \in B_{R}$. Therefore, by the Arzelá-Ascoli theorem, the operator $\mathbb{Q}: C([1, e], \mathbb{R}) \rightarrow$ $C([1, e], \mathbb{R})$ is completely continuous.

Let $x$ be a solution. Then, for $t \in[1, e]$, and following similar computations to the first step, we have

$$
|x(t)| \leq\|\rho\| \Upsilon(\|x\|) \Lambda(\alpha)+|\lambda|\|x\| \Lambda(0),
$$

which leads to

$$
\frac{\|x\|}{\|\rho\| \Upsilon(\|x\|) \Lambda(\alpha)+|\lambda|\|x\| \Lambda(0)} \leq 1 .
$$

In view of $\left(H_{4}\right)$, there exists $M$ such that $\|x\| \neq M$. Let us set

$$
U=\{x \in C([1, e], \mathbb{R}):\|x\|<M\} .
$$

We see that the operator $Q: \bar{U} \rightarrow C([1, e], \mathbb{R})$ is continuous and completely continuous. From the choice of $U$, there is no $x \in \partial U$ such that $x=\xi Q x$ for some $\xi \in$ $(0,1)$. Consequently, by the nonlinear alternative of LeraySchauder's type, we deduce that $Q$ has a fixed point $x \in \bar{U}$ which is a solution of the boundary value problem (1). This completes the proof.

\subsection{Existence Result via Leray-Schauder's Degree Theory}

Theorem 10. Let $f:[1, e] \times \mathbb{R} \rightarrow \mathbb{R}$ be a continuous function. In addition, one assumes the following:

$\left(H_{5}\right)$ there exist constants $0 \leq \mu<[1-|\lambda| \Lambda(0)][\Lambda(\alpha)]^{-1}$ and $M>0$ such that

$$
|f(t, x)| \leq \mu|x|+M \quad \forall(t, x) \in[1, e] \times \mathbb{R},
$$

where $\Lambda(0)$ and $\Lambda(\alpha)$ are given by (18) with $u=0$ and $\alpha$, respectively.

Then, the nonlocal problem (1) has at least one solution on $[1, e]$.

Proof. We define an operator $Q$ : $C([1, e], \mathbb{R}) \rightarrow C([1, e], \mathbb{R})$ as in (17) and consider the fixed point problem

$$
x=Q x
$$

We are going to prove that there exists a fixed point $x \in$ $C([1, e], \mathbb{R})$ satisfying (39). It is sufficient to show that $\mathbb{Q}$ : $\bar{B}_{R} \rightarrow C([1, e], \mathbb{R})$ satisfies

$$
x \neq \kappa Q \mathcal{Q} x, \quad \forall x \in \partial B_{R}, \forall \kappa \in[0,1],
$$


where $B_{R}=\left\{x \in C([1, e], \mathbb{R}): \max _{t \in[1, e]}|x(t)|<R, R>0\right\}$. We define

$$
H(\kappa, x)=\kappa(\mathbb{Q} x, \quad x \in C([1, e], \mathbb{R}), \kappa \in[0,1] .
$$

As shown in Theorem 9, the operator $\mathbb{Q}$ is continuous, uniformly bounded, and equicontinuous. Then, by the ArzeláAscoli theorem, a continuous map $h_{\kappa}$ defined by $h_{\kappa}(x)=$ $x-H(\kappa, x)=x-\kappa Q \mathbb{Q} x$ is completely continuous. If $(40)$ is true, then the following Leray-Schauder degrees are well defined and, by the homotopy invariance of topological degree, it follows that

$$
\begin{aligned}
\operatorname{deg}\left(h_{\kappa}, B_{R}, 0\right) & =\operatorname{deg}\left(I-\kappa \mathscr{Q}, B_{R}, 0\right)=\operatorname{deg}\left(h_{1}, B_{R}, 0\right) \\
& =\operatorname{deg}\left(h_{0}, B_{R}, 0\right)=\operatorname{deg}\left(I, B_{R}, 0\right) \\
& =1 \neq 0, \quad 0 \in B_{R},
\end{aligned}
$$

where $I$ denotes the identity operator. By the nonzero property of Leray-Schauder's degree, $h_{1}(x)=x-\mathbb{Q} x=0$ for at least one $x \in B_{R}$. In order to prove (40), we assume that $x=\kappa \mathbb{Q} x$ for some $\kappa \in[0,1]$. Then

$$
\begin{aligned}
& |(Q, x)(t)| \\
& \leq \mid \frac{1}{\Omega}\left[\left(\Omega_{4}-\frac{(\log t)^{\beta}}{\Gamma(\beta+1)} \Omega_{3}\right)\right. \\
& \quad \times\left(\sum _ { j = 1 } ^ { n } \phi _ { j } \left[I^{\alpha+\beta+\gamma_{j}} f(s, x(s))\left(\omega_{j}\right)\right.\right. \\
& \left.-\lambda I^{\beta+\gamma_{j}} x(s)\left(\omega_{j}\right)\right] \\
& -\sum_{i=1}^{m} \theta_{i}\left[I^{\alpha+\beta+\mu_{i}} f(s, x(s))\left(\eta_{i}\right)\right. \\
& +\left(\frac{(\log t)^{\beta}}{\Gamma(\beta+1)} \Omega_{1}-\Omega_{2}\right) \\
& \times\left(\sum_{l=1}^{q} \nu_{l}\left[I^{\alpha+\beta+\mu_{i}} x(s)\left(\eta_{i}\right)\right]\right) \\
& +I^{\alpha+\beta} f(s, x(s))(t)-\lambda I^{\beta} x(s)(t) \mid \\
& \left.-\lambda I^{\beta+\tau_{l}} x(s)\left(\varphi_{l}\right)\right] \\
& -\sum_{k=1}^{p} \varepsilon_{k}\left[I^{\alpha+\beta+\sigma_{k}} f(s, x(s))\left(\psi_{k}\right)\right. \\
& \left.\left.\left.-\lambda I^{\beta+\sigma_{k}} x(s)\left(\psi_{k}\right)\right]\right)\right]
\end{aligned}
$$

$$
\begin{aligned}
& \leq \frac{1}{|\Omega|}\left[\left(\left|\Omega_{4}\right|+\frac{(\log t)^{\beta}}{\Gamma(\beta+1)}\left|\Omega_{3}\right|\right)\right. \\
& \times\left(\sum _ { j = 1 } ^ { n } | \phi _ { j } | \left[I^{\alpha+\beta+\gamma_{j}}|f(s, x(s))|\left(\omega_{j}\right)\right.\right. \\
& \left.+|\lambda| I^{\beta+\gamma_{j}}|x(s)|\left(\omega_{j}\right)\right] \\
& +\sum_{i=1}^{m}\left|\theta_{i}\right|\left[I^{\alpha+\beta+\mu_{i}}|f(s, x(s))|\left(\eta_{i}\right)\right. \\
& \left.\left.+|\lambda| I^{\beta+\mu_{i}}|x(s)|\left(\eta_{i}\right)\right]\right) \\
& +\left(\frac{(\log t)^{\beta}}{\Gamma(\beta+1)}\left|\Omega_{1}\right|+\left|\Omega_{2}\right|\right) \\
& \times\left(\sum _ { l = 1 } ^ { q } | v _ { l } | \left[I^{\alpha+\beta+\tau_{l}}|f(s, x(s))|\left(\varphi_{l}\right)\right.\right. \\
& \left.+|\lambda| I^{\beta+\tau_{l}}|x(s)|\left(\varphi_{l}\right)\right] \\
& +\sum_{k=1}^{p}\left|\varepsilon_{k}\right|\left[I^{\alpha+\beta+\sigma_{k}}|f(s, x(s))|\left(\psi_{k}\right)\right. \\
& \left.\left.\left.+|\lambda| I^{\beta+\sigma_{k}}|x(s)|\left(\psi_{k}\right)\right]\right)\right] \\
& +I^{\alpha+\beta}|f(s, x(s))|(t)+|\lambda| I^{\beta}|x(s)|(t) \\
& \leq(\mu\|x\|+M) \Lambda(\alpha)+|\lambda|\|x\| \Lambda(0) \\
& =[\mu \Lambda(\alpha)+|\lambda| \Lambda(0)]\|x\|+M \Lambda(\alpha) .
\end{aligned}
$$

Computing directly for $\|x\|=\sup _{t \in[1, e]}|x(t)|$, we have

$$
\|x\| \leq \frac{M \Lambda(\alpha)}{1-\mu \Lambda(\alpha)-|\lambda| \Lambda(0)} .
$$

If $R=M \Lambda(\alpha) /(1-\mu \Lambda(\alpha)-|\lambda| \Lambda(0))+1$, inequality (39) holds. This completes the proof.

\section{Examples}

In this section, we present some examples to illustrate our results. 
Example 1. Consider the following nonlinear Langevin equation of Hadamard-Caputo type fractional derivatives with nonlocal fractional integral conditions:

$$
\begin{gathered}
D^{1 / 2}\left(D^{2 / 3}+\frac{1}{9}\right) x(t)=\frac{\log t}{3 t} \cdot \frac{|x(t)|}{2+|x(t)|}+\frac{3}{2} t, \quad 1<t<e, \\
\frac{13}{4} I^{2 / 3} x\left(\frac{e+7}{7}\right)+\frac{13}{3} I^{3 / 4} x\left(\frac{2 e+7}{7}\right)+\frac{13}{2} I^{4 / 5} x\left(\frac{3 e+7}{7}\right) \\
=\frac{1}{3} I^{1 / 6} x\left(\frac{e+2}{4}\right)+\frac{1}{4} I^{1 / 2} x\left(\frac{e+1}{2}\right), \\
\frac{2}{3} I^{1 / 5} x\left(\frac{3 e}{4}\right)+\frac{3}{7} I^{1 / 4} x\left(\frac{4 e}{5}\right)=3 I^{1 / 5} x\left(\frac{2 e}{5}\right) .
\end{gathered}
$$

Here, $\alpha=1 / 2, \beta=2 / 3, \lambda=1 / 9, m=3, n=2, p=2$, $q=1, \theta_{1}=13 / 4, \theta_{2}=13 / 3, \theta_{3}=13 / 2, \mu_{1}=2 / 3, \mu_{2}=3 / 4$, $\mu_{3}=4 / 5, \eta_{1}=(e+7) / 7, \eta_{2}=(2 e+7) / 7, \eta_{3}=(3 e+7) / 7$, $\phi_{1}=1 / 3, \phi_{2}=1 / 4, \gamma_{1}=1 / 6, \gamma_{2}=1 / 2, \omega_{1}=(e+2) / 4$, $\omega_{2}=(e+1) / 2, \varepsilon_{1}=2 / 3, \varepsilon_{2}=3 / 7, \sigma_{1}=1 / 5, \sigma_{2}=1 / 4$, $\psi_{1}=3 e / 4, \psi_{2}=4 e / 5, \nu_{1}=3, \tau_{1}=1 / 5, \varphi_{1}=2 e / 5$, and $f(t, x)=(\log t|x(t)|) /(3 t(2+|x(t)|))+3 t / 2$. Since $\mid f(t, x)-$ $f(t, y)|\leq(1 / 6)| x-y \mid$, then $\left(H_{1}\right)$ is satisfied with $L=1 / 6$. We can show that

$$
\begin{aligned}
\Lambda(\alpha)=\frac{1}{|\Omega|}[ & \left(\left|\Omega_{4}\right|+\frac{\left|\Omega_{3}\right|}{\Gamma(\beta+1)}\right) \\
& \times\left(\sum_{j=1}^{n}\left|\phi_{j}\right| \frac{\left(\log \omega_{j}\right)^{\alpha+\beta+\gamma_{j}}}{\Gamma\left(\alpha+\beta+\gamma_{j}+1\right)}\right. \\
& \left.+\left(\frac{\left|\Omega_{1}\right|}{\Gamma(\beta+1)}+\left|\Omega_{2}\right|\right) \frac{\left(\log \eta_{i}\right)^{\alpha+\beta+\mu_{i}}}{\Gamma\left(\alpha+\beta+\mu_{i}+1\right)}\right) \\
& \times\left(\sum_{l=1}^{q}\left|\nu_{l}\right| \frac{\left(\log \varphi_{l}\right)^{\alpha+\beta+\tau_{l}}}{\Gamma\left(\alpha+\beta+\tau_{l}+1\right)}\right. \\
+\frac{1}{\Gamma(\alpha+\beta+1)} & \left.\left.+\sum_{k=1}^{p}\left|\varepsilon_{k}\right| \frac{\left(\log \psi_{k}\right)^{\alpha+\beta+\sigma_{k}}}{\Gamma\left(\alpha+\beta+\sigma_{k}+1\right)}\right)\right]
\end{aligned}
$$$$
\approx 2.527538367 \text {, }
$$

$$
\begin{aligned}
\Lambda(0)=\frac{1}{|\Omega|}\left[\left(\left|\Omega_{4}\right|+\frac{\left|\Omega_{3}\right|}{\Gamma(\beta+1)}\right)\right. \\
\quad \times\left(\sum_{j=1}^{n}\left|\phi_{j}\right| \frac{\left(\log \omega_{j}\right)^{\beta+\gamma_{j}}}{\Gamma\left(\beta+\gamma_{j}+1\right)}\right. \\
\left.+\sum_{i=1}^{m}\left|\theta_{i}\right| \frac{\left(\log \eta_{i}\right)^{\beta+\mu_{i}}}{\Gamma\left(\beta+\mu_{i}+1\right)}\right)
\end{aligned}
$$

$$
\begin{aligned}
& +\left(\frac{\left|\Omega_{1}\right|}{\Gamma(\beta+1)}+\left|\Omega_{2}\right|\right) \\
& \times\left(\sum_{l=1}^{q}\left|\nu_{l}\right| \frac{\left(\log \varphi_{l}\right)^{\beta+\tau_{l}}}{\Gamma\left(\beta+\tau_{l}+1\right)}\right. \\
& \left.\left.\quad+\sum_{k=1}^{p}\left|\varepsilon_{k}\right| \frac{\left(\log \psi_{k}\right)^{\beta+\sigma_{k}}}{\Gamma\left(\beta+\sigma_{k}+1\right)}\right)\right]+\frac{1}{\Gamma(\beta+1)}
\end{aligned}
$$$$
\approx 4.083246365 \text {. }
$$

Thus, $L \Lambda(\alpha)+|\lambda| \Lambda(0) \approx 0.8749504351<1$. Hence, by Theorem 5 , the nonlocal problem (45) has a unique solution on $[1, e]$.

Example 2. Consider the following nonlinear Langevin equation of Hadamard-Caputo type fractional derivatives with nonlocal fractional integral conditions:

$$
\begin{aligned}
& D^{2 / 3}\left(D^{2 / 5}+\frac{1}{12}\right) x(t) \\
&= \frac{t \log t}{t+\sin \pi t} \cdot \frac{\operatorname{arccot} x(t)}{1+|x(t)|}+t^{2} \sin \pi t, \quad 1<t<e, \\
& 4 I^{2 / 3} x\left(\frac{2 e}{3}\right)+3 I^{3 / 5} x\left(\frac{2 e+1}{3}\right) \\
&=\frac{2}{5} I^{2 / 3} x\left(\frac{e+1}{3}\right)+\frac{1}{2} I^{1 / 2} x\left(\frac{e+2}{3}\right) \\
& \quad+\frac{2}{5} I^{3 / 2} x\left(\frac{e+3}{3}\right), \\
& \frac{5}{7} I^{2 / 5} x\left(\frac{3 e}{7}\right)+\frac{6}{7} I^{3 / 4} x\left(\frac{4 e}{7}\right) \\
&=\frac{1}{5} I^{1 / 5} x\left(\frac{3 e}{5}\right)+\frac{2}{7} I^{2 / 5} x\left(\frac{4 e}{5}\right) .
\end{aligned}
$$

Here, $\alpha=2 / 3, \beta=2 / 5, \lambda=1 / 12, m=2, n=3, p=2$, $q=2, \theta_{1}=4, \theta_{2}=3, \mu_{1}=2 / 3, \mu_{2}=3 / 5, \eta_{1}=2 e / 3, \eta_{2}=$ $(2 e+1) / 3, \phi_{1}=2 / 5, \phi_{2}=1 / 2, \phi_{3}=2 / 5, \gamma_{1}=2 / 3, \gamma_{2}=1 / 2$, $\gamma_{3}=3 / 2, \omega_{1}=(e+1) / 3, \omega_{2}=(e+2) / 3, \omega_{3}=(e+3) / 3, \varepsilon_{1}=5 / 7$, $\varepsilon_{2}=6 / 7, \sigma_{1}=2 / 5, \sigma_{2}=3 / 4, \psi_{1}=3 e / 7, \psi_{2}=4 e / 7, \nu_{1}=1 / 5$, $\nu_{2}=2 / 7, \tau_{1}=1 / 5, \tau_{2}=2 / 5, \varphi_{1}=3 e / 5, \varphi_{2}=4 e / 5$, and $f(t, x)=(t \log t \operatorname{arccot} x(t)) /((t+\sin \pi t)(1+|x(t)|))+t^{2} \sin \pi t$. Since $|f(t, x)| \leq(t \log t) /(t+\sin \pi t)+t^{2} \sin \pi t$, then $\left(H_{2}\right)$ is satisfied. We can find that

$$
\begin{aligned}
\Lambda(0)=\frac{1}{|\Omega|}\left[\left(\left|\Omega_{4}\right|+\frac{\left|\Omega_{3}\right|}{\Gamma(\beta+1)}\right)\right. \\
\quad \times\left(\sum_{j=1}^{n}\left|\phi_{j}\right| \frac{\left(\log \omega_{j}\right)^{\beta+\gamma_{j}}}{\Gamma\left(\beta+\gamma_{j}+1\right)}\right. \\
\left.+\sum_{i=1}^{m}\left|\theta_{i}\right| \frac{\left(\log \eta_{i}\right)^{\beta+\mu_{i}}}{\Gamma\left(\beta+\mu_{i}+1\right)}\right)
\end{aligned}
$$




$$
\begin{aligned}
& +\left(\frac{\left|\Omega_{1}\right|}{\Gamma(\beta+1)}+\left|\Omega_{2}\right|\right) \\
& \times\left(\sum_{l=1}^{q}\left|\nu_{l}\right| \frac{\left(\log \varphi_{l}\right)^{\beta+\tau_{l}}}{\Gamma\left(\beta+\tau_{l}+1\right)}\right. \\
& \left.\left.\quad+\sum_{k=1}^{p}\left|\varepsilon_{k}\right| \frac{\left(\log \psi_{k}\right)^{\beta+\sigma_{k}}}{\Gamma\left(\beta+\sigma_{k}+1\right)}\right)\right]+\frac{1}{\Gamma(\beta+1)}
\end{aligned}
$$$$
\approx 10.69222877 \text {. }
$$

Thus, $|\lambda| \Lambda(0) \approx 0.8910190640<1$. Hence, by Theorem 7 , the nonlocal problem (47) has at least one solution on $[1, e]$.

Example 3. Consider the following nonlinear Langevin equation of Hadamard-Caputo type fractional derivatives with nonlocal fractional integral conditions:

$$
\begin{aligned}
& D^{1 / 2}\left(D^{4 / 5}+\frac{1}{7}\right) x(t) \\
& =\frac{\sin x(t)}{5 \pi^{2}+\cos ^{2} \pi x(t)}+\frac{2+\log t}{\pi^{2}}, \quad 1<t<e, \\
& 2 I^{3 / 2} x\left(\frac{e+1}{3}\right)+3 I^{4 / 3} x\left(\frac{e+2}{3}\right)+4 I^{5 / 4} x\left(\frac{e+3}{3}\right) \\
& \quad+5 I^{6 / 5} x\left(\frac{e+4}{3}\right)=\frac{2}{3} I^{2 / 3} x\left(\frac{e+3}{4}\right), \\
& \frac{1}{3} I^{3 / 5} x\left(\frac{2 e}{5}\right)+\frac{1}{6} I^{3 / 4} x\left(\frac{3 e}{5}\right) \\
& =5 I^{3 / 5} x\left(\frac{2 e}{5}\right)+\frac{1}{2} I^{2 / 5} x\left(\frac{3 e}{5}\right) .
\end{aligned}
$$

Here, $\alpha=1 / 2, \beta=4 / 5, \lambda=1 / 7, m=4, n=1, p=2$, $q=2, \theta_{1}=2, \theta_{2}=3, \theta_{3}=4, \theta_{4}=5, \mu_{1}=3 / 2, \mu_{2}=4 / 3$, $\mu_{3}=5 / 4, \mu_{4}=6 / 5, \eta_{1}=(e+1) / 3, \eta_{2}=(e+2) / 3, \eta_{3}=(e+3) / 3$, $\eta_{4}=(e+4) / 3, \phi_{1}=2 / 3, \gamma_{1}=2 / 3, \omega_{1}=(e+3) / 4, \varepsilon_{1}=1 / 3$, $\varepsilon_{2}=1 / 6, \sigma_{1}=3 / 5, \sigma_{2}=3 / 4, \psi_{1}=2 e / 5, \psi_{2}=3 e / 5, v_{1}=5$, $v_{2}=1 / 2, \tau_{1}=3 / 5, \tau_{2}=2 / 5, \varphi_{1}=2 e / 5, \varphi_{2}=3 e / 5$, and $f(t, x)=(\sin x(t)) /\left(5 \pi^{2}+\cos ^{2} \pi x(t)\right)+(2+\log t) / \pi^{2}$. Then, we get that

$$
\begin{aligned}
\Lambda(\alpha)=\frac{1}{|\Omega|}\left[\left(\left|\Omega_{4}\right|+\frac{\left|\Omega_{3}\right|}{\Gamma(\beta+1)}\right)\right. \\
\quad \times\left(\sum_{j=1}^{n}\left|\phi_{j}\right| \frac{\left(\log \omega_{j}\right)^{\alpha+\beta+\gamma_{j}}}{\Gamma\left(\alpha+\beta+\gamma_{j}+1\right)}\right. \\
+\left(\frac{\left|\Omega_{1}\right|}{\Gamma(\beta+1)}+\left|\Omega_{2}\right|\right)
\end{aligned}
$$

$$
\begin{gathered}
\times\left(\sum_{l=1}^{q}\left|\nu_{l}\right| \frac{\left(\log \varphi_{l}\right)^{\alpha+\beta+\tau_{l}}}{\Gamma\left(\alpha+\beta+\tau_{l}+1\right)}\right. \\
\left.\left.+\sum_{k=1}^{p}\left|\varepsilon_{k}\right| \frac{\left(\log \psi_{k}\right)^{\alpha+\beta+\sigma_{k}}}{\Gamma\left(\alpha+\beta+\sigma_{k}+1\right)}\right)\right] \\
+\frac{1}{\Gamma(\alpha+\beta+1)} \\
\approx 2.675517413,
\end{gathered}
$$

$$
\begin{aligned}
\Lambda(0)=\frac{1}{|\Omega|}[ & \left(\left|\Omega_{4}\right|+\frac{\left|\Omega_{3}\right|}{\Gamma(\beta+1)}\right) \\
& \times\left(\sum_{j=1}^{n}\left|\phi_{j}\right| \frac{\left(\log \omega_{j}\right)^{\beta+\gamma_{j}}}{\Gamma\left(\beta+\gamma_{j}+1\right)}\right. \\
& \left.+\sum_{i=1}^{m}\left|\theta_{i}\right| \frac{\left(\log \eta_{i}\right)^{\beta+\mu_{i}}}{\Gamma\left(\beta+\mu_{i}+1\right)}\right) \\
& +\left(\frac{\left|\Omega_{1}\right|}{\Gamma(\beta+1)}+\left|\Omega_{2}\right|\right) \\
& \times\left(\sum_{l=1}^{q}\left|\nu_{l}\right| \frac{\left(\log \varphi_{l}\right)^{\beta+\tau_{l}}}{\Gamma\left(\beta+\tau_{l}+1\right)}\right. \\
& \left.\left.+\sum_{k=1}^{p}\left|\varepsilon_{k}\right| \frac{\left(\log \psi_{k}\right)^{\beta+\sigma_{k}}}{\Gamma\left(\beta+\sigma_{k}+1\right)}\right)\right]+\frac{1}{\Gamma(\beta+1)}
\end{aligned}
$$$$
\approx 5.058796431 \text {. }
$$

Clearly,

$$
\begin{aligned}
|f(t, x)| & =\left|\frac{\sin x(t)}{5 \pi^{2}+\cos ^{2} \pi x(t)}+\frac{2+\log t}{\pi^{2}}\right| \\
& \leq\left(\frac{2+\log t}{5 \pi^{2}}\right)(|x(t)|+5) .
\end{aligned}
$$

Choosing $\rho(t)=(2+\log t) /\left(5 \pi^{2}\right)$ and $\Upsilon(|x|)=|x|+5$, we can show that

$$
\frac{M}{\|\rho\| \Upsilon(M) \Lambda(\alpha)+|\lambda| M \Lambda(0)}>1
$$

which implies that $M>7.092618387$. Hence, by Theorem 9, the nonlocal problem (49) has at least one solution on $[1, e]$.

\section{Conflict of Interests}

The authors declare that there is no conflict of interests regarding the publication of this paper. 


\section{Acknowledgment}

This research was funded by King Mongkut's University of Technology North Bangkok (Contract no. KMUTNB-GEN57-21).

\section{References}

[1] S. G. Samko, A. A. Kilbas, and O. I. Marichev, Fractional Integrals and Derivatives, Theory and Applications, Gordon and Breach Science, Yverdon, Switzerland, 1993.

[2] I. Podlubny, Fractional Differential Equations, Academic Press, San Diego, Calif, USA, 1999.

[3] A. A. Kilbas, H. M. Srivastava, and J. J. Trujillo, Theory and Applications of Fractional Differential Equations, North-Holland Mathematics Studies, 204, Elsevier Science B.V., Amsterdam, The Netherlands, 2006.

[4] D. Baleanu, K. Diethelm, E. Scalas, and J. J. Trujillo, Fractional Calculus Models and Numerical Methods, Complexity, Nonlinearity and Chaos, World Scientific, Boston, Mass, USA, 2012.

[5] R. P. Agarwal, Y. Zhou, and Y. He, "Existence of fractional neutral functional differential equations," Computers \& Mathematics with Applications, vol. 59, no. 3, pp. 1095-1100, 2010.

[6] D. Băleanu, O. G. Mustafa, and R. P. Agarwal, "On $L^{P}$-solutions for a class of sequential fractional differential equations," Applied Mathematics and Computation, vol. 218, no. 5, pp. 20742081, 2011.

[7] B. Ahmad and J. J. Nieto, "Riemann-Liouville fractional integrodifferential equations with fractional nonlocal integral boundary conditions," Boundary Value Problems, vol. 2011, article 36, p. 9, 2011.

[8] B. Ahmad, S. K. Ntouyas, and A. Alsaedi, "New existence results for nonlinear fractional differential equations with threepoint integral boundary conditions," Advances in Difference Equations, vol. 2011, Article ID 107384, 11 pages, 2011.

[9] D. O’Regan and S. Staněk, "Fractional boundary value problems with singularities in space variables," Nonlinear Dynamics, vol. 71, no. 4, pp. 641-652, 2013.

[10] B. Ahmad, S. K. Ntouyas, and A. Alsaedi, "A study of nonlinear fractional differential equations of arbitrary order with Riemann-Liouville type multistrip boundary conditions," Mathematical Problems in Engineering, vol. 2013, Article ID 320415, 9 pages, 2013.

[11] B. Ahmad and J. J. Nieto, "Boundary value problems for a class of sequential integrodifferential equations of fractional order," Journal of Function Spaces and Applications, vol. 2013, Article ID 149659, 8 pages, 2013.

[12] L. Zhang, B. Ahmad, G. Wang, and R. P. Agarwal, "Nonlinear fractional integro-differential equations on unbounded domains in a Banach space," Journal of Computational and Applied Mathematics, vol. 249, pp. 51-56, 2013.

[13] X. Liu, M. Jia, and W. Ge, "Multiple solutions of a p-Laplacian model involving a fractional derivative," Advances in Difference Equations, vol. 2013, article 126, 2013.

[14] J. Hadamard, "Essai sur l'etude des fonctions donnees par leur developpment de Taylor," Journal de Mathématiques Pures et Appliquées, vol. 8, pp. 101-186, 1892.

[15] P. L. Butzer, A. A. Kilbas, and J. J. Trujillo, "Compositions of Hadamard-type fractional integration operators and the semigroup property," Journal of Mathematical Analysis and Applications, vol. 269, no. 2, pp. 387-400, 2002.
[16] P. L. Butzer, A. A. Kilbas, and J. J. Trujillo, "Fractional calculus in the Mellin setting and Hadamard-type fractional integrals," Journal of Mathematical Analysis and Applications, vol. 269, no. 1, pp. 1-27, 2002.

[17] P. L. Butzer, A. A. Kilbas, and J. J. Trujillo, "Mellin transform analysis and integration by parts for Hadamard-type fractional integrals," Journal of Mathematical Analysis and Applications, vol. 270, no. 1, pp. 1-15, 2002.

[18] A. A. Kilbas, "Hadamard-type fractional calculus," Journal of the Korean Mathematical Society, vol. 38, no. 6, pp. 1191-1204, 2001.

[19] A. A. Kilbas and J. J. Trujillo, "Hadamard-type integrals as Gtransforms," Integral Transforms and Special Functions, vol. 14, no. 5, pp. 413-427, 2003.

[20] W. T. Coffey, Y. P. Kalmykov, and J. T. Waldron, The Langevin Equation, World Scientific, Singapore, 2nd edition, 2004.

[21] S. C. Lim, M. Li, and L. P. Teo, "Langevin equation with two fractional orders," Physics Letters A, vol. 372, no. 42, pp. 63096320, 2008.

[22] S. C. Lim and L. P. Teo, "The fractional oscillator process with two indices," Journal of Physics A: Mathematical and Theoretical, vol. 42, no. 6, Article ID 065208, 34 pages, 2009.

[23] M. Uranagase and T. Munakata, "Generalized Langevin equation revisited: mechanical random force and self-consistent structure," Journal of Physics. A. Mathematical and Theoretical, vol. 43, no. 45, Article ID 455003, 11 pages, 2010.

[24] S. I. Denisov, H. Kantz, and P. Hänggi, "Langevin equation with super-heavy-tailed noise," Journal of Physics A, vol. 43, no. 28, Article ID 285004, 2010.

[25] A. Lozinski, R. G. Owens, and T. N. Phillips, "The langevin and fokker-planck equations in polymer rheology," Handbook of Numerical Analysis, vol. 16, pp. 211-303, 2011.

[26] L. Lizana, T. Ambjörnsson, A. Taloni, E. Barkai, and M. A. Lomholt, "Foundation of fractional Langevin equation: harmonization of a many-body problem," Physical Review E, vol. 81, Article ID 051118, 2010.

[27] Y. Y. Gambo, F. Jarad, D. Baleanu, and T. Abdeljawad, "On Caputo modification of the Hadamard fractional derivatives," Advances in Difference Equations, vol. 2014, article 10, 2014.

[28] B. Ahmad and P. Eloe, "A nonlocal boundary value problem for a nonlinear fractional differential equation with two indices," Communications on Applied Nonlinear Analysis, vol. 17, no. 3, pp. 69-80, 2010.

[29] B. Ahmad, J. J. Nieto, A. Alsaedi, and M. El-Shahed, "A study of nonlinear Langevin equation involving two fractional orders in different intervals," Nonlinear Analysis: Real World Applications, vol. 13, no. 2, pp. 599-606, 2012.

[30] J. R. Cannon, "The solution of the heat equation subject to the specification of energy," Quarterly of Applied Mathematics, vol. 21, pp. 155-160, 1963.

[31] A. Bouziani, "On the solvability of nonlocal pluriparabolic problems," Electronic Journal of Differential Equations, vol. 21, pp. 1-16, 2001.

[32] S. Mesloub and S. A. Messaoudi, "A nonlocal mixed semilinear problem for second-order hyperbolic equations," Electronic Journal of Differential Equations, vol. 30, pp. 1-17, 2003.

[33] L. S. Pul'kina, "A nonlocal problem with integral conditions for a hyperbolic equation," Differential Equations, vol. 40, no. 7, pp. 947-953, 2004.

[34] G. Avalishvili, M. Avalishvili, and D. Gordeziani, "On integral nonlocal boundary value problems for some partial differential equations," Bulletin of the Georgian National Academy of Sciences, vol. 5, no. 1, pp. 31-37, 2011. 
[35] F. Jarad, T. Abdeljawad, and D. Baleanu, "Caputo-type modification of the Hadamard fractional derivatives," Advances in Difference Equations, vol. 2012, article 142, 2012.

[36] M. A. Krasnosel'skii, "Two remarks on the method of successive approximations," Uspekhi Matematicheskikh Nauk, vol. 10, no. 1, pp. 123-127, 1955.

[37] A. Granas and J. Dugundji, Fixed Point Theory, Springer, New York, NY, USA, 2003. 


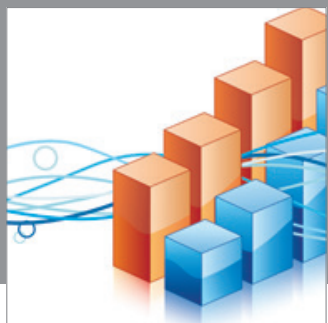

Advances in

Operations Research

mansans

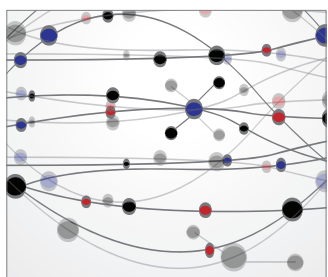

The Scientific World Journal
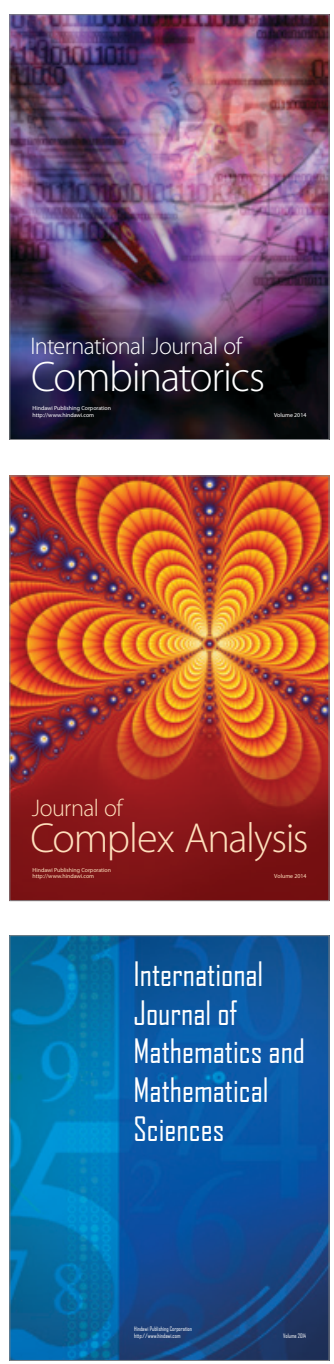
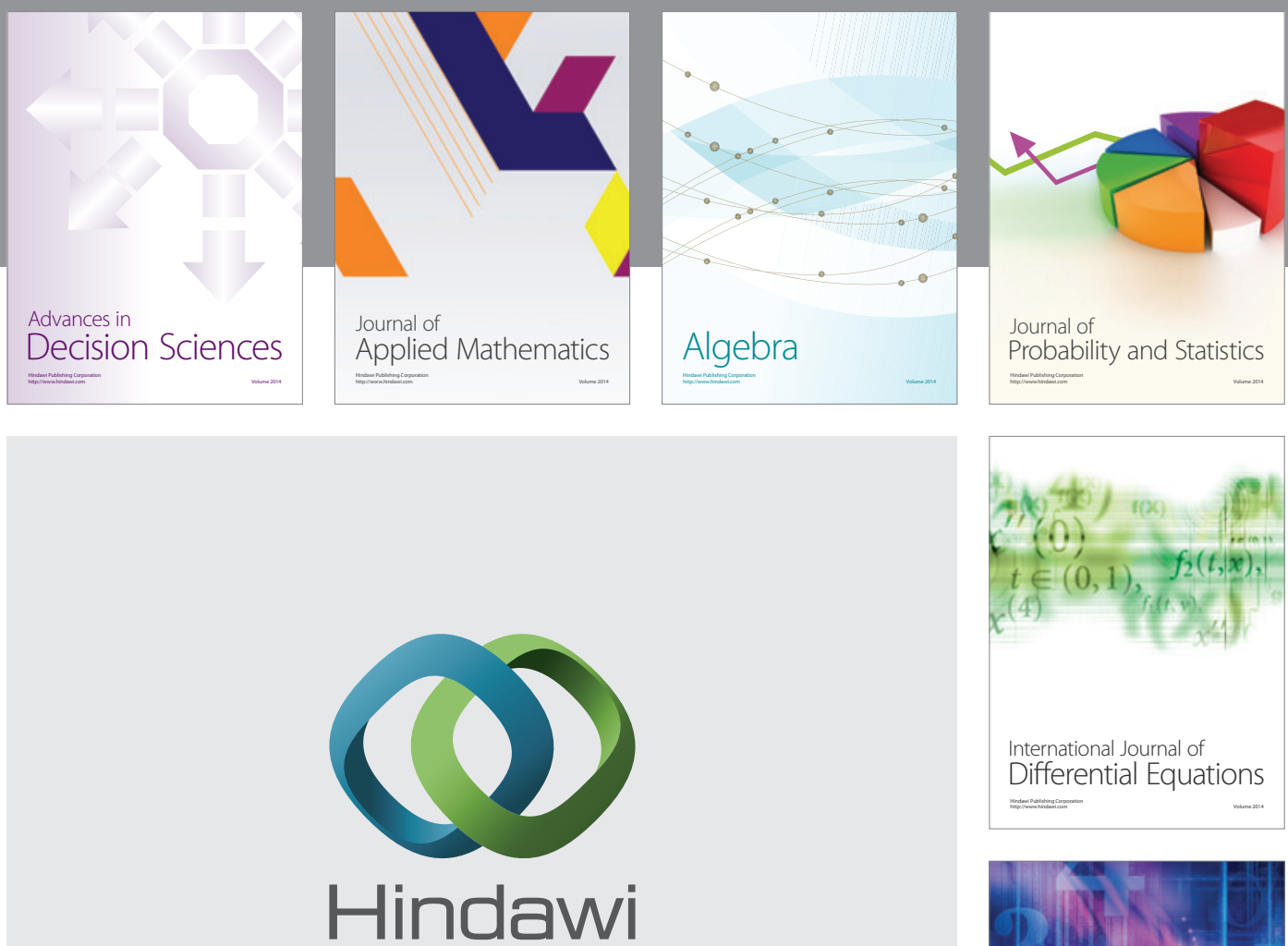

Submit your manuscripts at http://www.hindawi.com
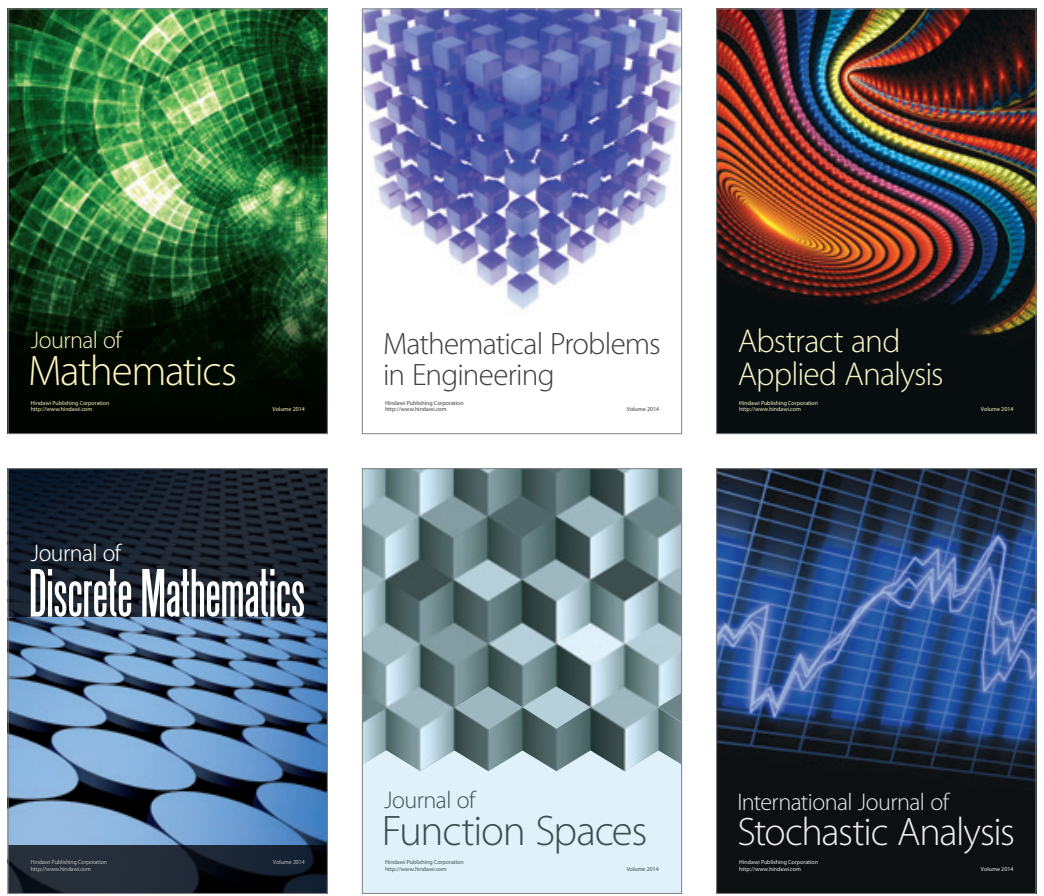

Journal of

Function Spaces

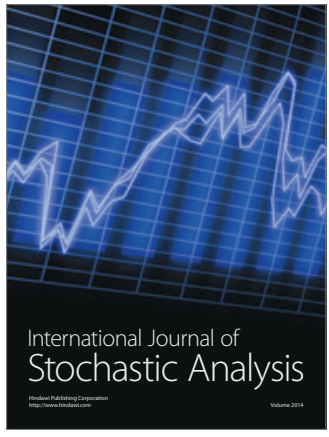

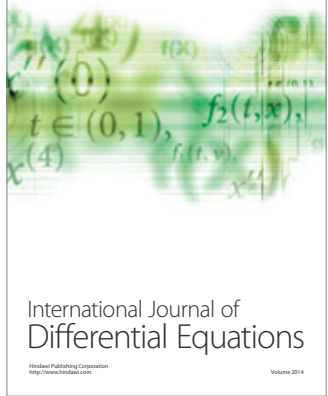
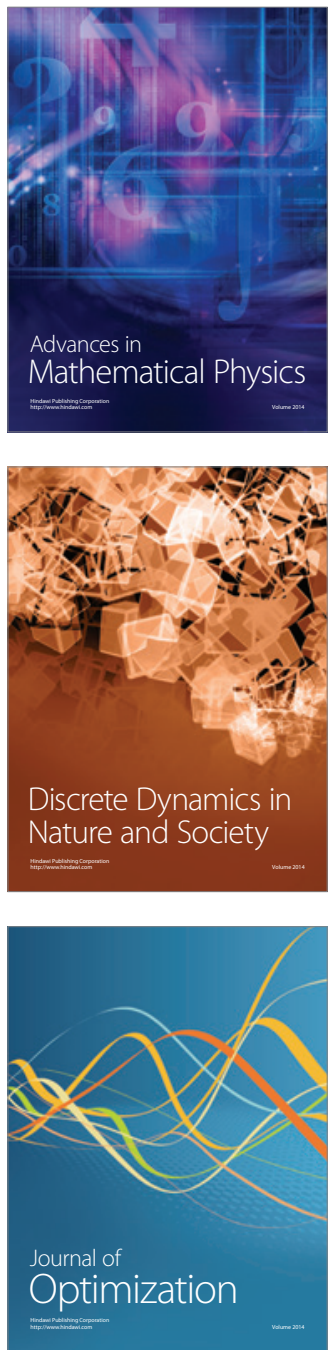